\title{
A posteriori analysis for dynamic model adaptation in convection dominated problems
}

Article

Accepted Version

Giesselmann, J. and Pryer, T. (2017) A posteriori analysis for dynamic model adaptation in convection dominated problems. Mathematical models and methods in applied Sciences (M3AS), 27 (13). pp. 2381-2423. ISSN 0218-2025 doi: https://doi.org/10.1142/S0218202517500476 Available at https://centaur.reading.ac.uk/71360/

It is advisable to refer to the publisher's version if you intend to cite from the work. See Guidance on citing.

To link to this article DOI: http://dx.doi.org/10.1142/S0218202517500476

Publisher: World Scientific

All outputs in CentAUR are protected by Intellectual Property Rights law, including copyright law. Copyright and IPR is retained by the creators or other copyright holders. Terms and conditions for use of this material are defined in the End User Agreement.

www.reading.ac.uk/centaur 
Central Archive at the University of Reading

Reading's research outputs online 


\title{
A POSTERIORI ANALYSIS FOR DYNAMIC MODEL ADAPTATION IN CONVECTION DOMINATED PROBLEMS
}

\author{
JAN GIESSELMANN AND TRISTAN PRYER
}

\begin{abstract}
In this work we present an a posteriori error indicator for approximation schemes of RungeKutta-discontinuous-Galerkin type arising in applications of compressible fluid flows. The purpose of this indicator is not only for mesh adaptivity, we also make use of this to drive model adaptivity. This is where a perhaps costly complex model and a cheaper simple model are solved over different parts of the domain. The a posteriori bound we derive indicates the regions where the complex model can be relatively well approximated with the cheaper one. One such example which we choose to highlight is that of the Navier-Stokes-Fourier equations approximated by Euler's equations.
\end{abstract}

\section{INTRODUCTION}

This paper is concerned with model adaptation in the context of advection dominated, compressible fluid flows as they appear in, for example, aerospace engineering. Compressible fluid flows can be described by different models having different levels of complexity. One example are the compressible Euler equations which are the limit of the Navier-Stokes-Fourier (NSF) equations when heat conduction and viscosity go to zero. Arguably the NSF system provides a more accurate description of reality since viscous effects which are neglected in Euler's equation play a dominant role in certain flow regimes like thin regions near obstacles, for example, aerofoils exhibiting Prandtls's boundary layers [Nic73]. However, viscous effects are negligible in large parts of the computational domain where convective effects dominate [BCR89, CW01, DGQ12]. Thus, it is desirable to avoid the effort of handling the viscous terms in these parts of the domain, that is, to use the NSF system only where needed and simpler models, e.g., (linearised) Euler equations, on the rest of the computational domain.

This insight has lead to the development, of a certain type, of so-called heterogeneous domain decomposition methods in which on a certain part of the computational domain the NSF equations are solved numerically, whereas the (linearised) Euler equations are used for far field computations [USDM06, CW01, Xu00, BHR11, e.g.]. In those works the domain was decomposed a priori, i.e., before the start of the numerical computation. The accuracy and efficiency of those schemes depends sensitively on the placement of the domains. Thus, the user is required to have some physical intuition on where to put the domain boundary.

It has been suggested that applying a more "adaptive" approach using cutoff functions to the dissipative terms in the NSF equations might lead to a modified model only containing second derivatives when a certain threshold is exceeded [BCR89, AP93]. The disadvantage with this approach is that error control for this type of model adaptation is not available, and the main justification for its use is that the modified equations converge to the original ones when the cut-off parameter tends to zero. A more rigorous model adaptation approach for stationary linear advection-diffusion systems based on optimal control techniques can be found in [AGQ06] and goal-oriented modelling error estimates for viscous and inviscid Burgers' equations were presented in [CBvB05]. Recently a heterogeneous domain decomposition technique for linear model problems based on factorisation was suggested in [GHM16].

Our approach to this issue differs from the previous ones, except [AGQ06] and [CBvB05] in that we aim at having an a posteriori criterion which enables an automatic and adaptive choice of domains. Our approach is inspired by [BE03] where a model adaptive algorithm based on an a posteriori modeling error estimator is presented. In [BE03] such an estimator was developed for hierarchies of elliptic models based on dual weighted residuals. This approach easily extends to parabolic problems and was extended to viscous, incompressible flows in [BE04, BBRR07, SRO11, vOBPvB15]. It should be noted that [BE04, SRO11, PV14, vOBPvB15] combine model adaptation with mesh adaptation. Earlier approaches to model adaptation based on a posteriori estimators or indicators are [ASS99, SO99, OV00, VO01].

Date: July 7, 2017.

We gratefully acknowledge that this work was supported by the German Research Foundation (DFG) via SFB TRR 75 'Tropfendynamische Prozesse unter extremen Umgebungsbedingungen'. TP also gratefully acknowledges the support of the EPSRC grant EP/P000835/1 "Adaptive Regularisation". 
The situation which we consider differs from the one studied in [BE03] since the problems at hand are highly nonlinear and convection dominated in nature. In particular, it is known that error estimators based on dual weighted residuals have certain theoretical limitations for nonlinear systems of hyperbolic conservation laws, e.g., Euler's equations, since the dual problems may become ill-posed in case the solution to the primal problem is discontinuous [HH02].

Our estimates are based on non-linear energy-like arguments using the relative entropy framework. A similar methodology was used in [Fis15] where rigorous a posteriori error estimates for the assumption of incompressibility in viscous flows were presented. It is similar to our approach in that it does not rely on the solution of dual problems but uses the relative entropy stability framework. Our presentation will focus on Runge-Kutta-discontinuous-Galerkin (RKDG) discretisations which are an established tool for viscous as well as inviscid compressible fluid flows. The schemes we study use discretisations of the NSF equations on some part of the computational domain and discretisations of Euler's equations on the rest of the domain. Following [GMP15, DG15], which were concerned with a posteriori estimators for discretisation errors for hyperbolic conservation laws, we define (explicitly computable) reconstructions of the numerical solution and use the relative entropy stability framework in order to derive an explicitly computable bound for the difference between the reconstruction and the exact solution to the NSF equations. Our error estimator, in fact, consists of two parts. One part is related to the modelling error while the other one is related to the numerical discretisation error such that our estimator allows for simultaneous model and mesh adaptation. Model adaptation for hyperbolic conservation laws and relaxation systems was addressed recently in [MCGS15]. In this work an error estimator for scalar problems, based on Kruzhkovs $\mathrm{L}_{1}$ stability theory, was presented. Additional relevant work is $\left[\mathrm{CCG}^{+} 16\right]$ where an error indicator for general systems based on Chapman-Enskog expansions was derived.

While the main motivation of our work stems from model adaptation between Euler and NSF equations and between isothermal Euler and isothermal Navier-Stokes equations, we present our analysis on a more abstract level, which we will describe in the next paragraph. We do this to allow the treatment of many cases simultaneously and, as such, it constitutes the central part of our analysis.

In the abstract framework we consider general models that include dissipative effects of the form:

$$
\partial_{t} \boldsymbol{u}+\sum_{\alpha} \partial_{x_{\alpha}} \boldsymbol{f}_{\alpha}(\boldsymbol{u})=\sum_{\alpha} \partial_{x_{\alpha}}\left(\varepsilon \boldsymbol{g}_{\alpha}(\boldsymbol{u}, \nabla \boldsymbol{u})\right) \text { on } \mathbb{T}^{d} \times(0, T)
$$

where $\boldsymbol{u}$ is the (unknown) vector of conserved quantities, $\varepsilon>0$ is a small parameter, $\boldsymbol{f}_{\alpha} \in \mathrm{C}^{2}\left(U, \mathbb{R}^{n}\right)$, $\alpha=1, \ldots, d$ are the components of an advective flux and $\boldsymbol{g}_{\alpha} \in \mathrm{C}^{2}\left(U \times \mathbb{R}^{d \times n}, \mathbb{R}^{n}\right), \alpha=1, \ldots, d$ are the components of a diffusive flux. Moreover, the so-called state space $U \subset \mathbb{R}^{n}$ is an open set; $T>0$ is some finite time and $\mathbb{T}^{d}$ denotes the $d$-dimensional flat torus.

We are interested in the case of $\varepsilon$ being very small, so that on large parts of the computational domain the inviscid model

$$
\partial_{t} \boldsymbol{u}+\sum_{\alpha} \partial_{x_{\alpha}} \boldsymbol{f}_{\alpha}(\boldsymbol{u})=0 \text { on } \mathbb{T}^{d} \times(0, T)
$$

is a reasonable approximation of (1.1), for which, numerical methods are computationally cheaper.

We will show later how the NSF equations fit into the framework (1.1) and that the Euler equations have the form (1.2). Indeed, we will present our analysis first for a scalar model problem, where we are able to derive fully computable a posteriori estimators. After treating the scalar case we advance to pairs of models (1.2) and (1.1), which also exist in applications beyond compressible fluid flows, for example traffic modelling.

We will assume throughout this work that (1.1) and (1.2) are endowed with an (identical) convex entropy/entropy flux pair. This assumption is true for the Euler and NSF model hierarchy, and expresses that both models are compatible with the second law of thermodynamics. For the scalar model problem it is trivially satisfied.

This entropy pair gives rise to a stability framework via the so-called relative entropy, which we will exploit in this work. The relative entropy framework for the NSF equations has received a lot of interest in recent years, e.g., [FJN12, LT13]. It enables us to study modelling errors for inviscid approximations of viscous, compressible flows in an a posteriori fashion. In the case $n=1$ and general $d$, which is the scalar case, we are able to prove rigorous a posteriori estimates. All constants are explicitly computable and we call such quantities estimators. When $n>1$ a non-computable constant appears in the argument which we were not able to circumvent. The resultant a posteriori bounds are called indicators in the sequel. Based on this a posteriori estimator/indicator we construct a model adaptive algorithm which we have implemented for model problems. 
There are many applications, e.g., aeroacoustics [USDM06], where it would be desirable to consider the pair of models consisting of NSF and the linearised Euler equations for computational efficiency. We are currently unable to deal with this setting since our analysis relies heavily on (1.1) and (1.2) having the same entropy functional, while the linearised Euler system has a different entropy.

A posteriori analysis is always based on the stability framework of the underlying problem. For the class of problems considered here we make use of the relative entropy framework which requires that (at least) one of the solutions is Lipschitz continuous in space. In principle, the model adaptation idea is independent of the numerical discretisations of both models, but for most approximation schemes for compressible fluid flows (finite volumes, discontinuous Galerkin) the discrete solution itself is not smooth enough to be used in the relative entropy stability analysis. Thus, our a posteriori argument requires a reconstruction approach. We will describe this reconstruction in detail for $\mathrm{dG}$ schemes. Note that, in principle, our a posteriori analysis gives rise to a modelling error indicator in a wide variety of numerical schemes. The bound relies heavily on a computable, Lipschitz continuous approximation of the exact solution, but it does not matter whether this results from a continuous finite element scheme of Taylor-Hood type, for example, or a reconstruction of a finite volume or dG solution.

The outline of this paper is as follows: In $\S 2$ we present the general framework of designing a posteriori estimators based on the study of the abstract equations (1.1)-(1.2) in the scalar case. In $\S 3$ we establish some structural properties of the INS and NSF systems regarding the compatibility of their diffusion terms with their relative entropies. In $\S 4$ we describe how the relative entropy framework can be extended to those generic pairs of systems of the form (1.1) and (1.2) that satisfy the structural properties that we established for the specific example of the NSF/Euler problem in $\S 3$. In $\S 5$ we give an overview of the reconstruction approach presented in [GMP15, DG15]. We also take the opportunity to extend the operators to 2 spatial dimensions for use in numerical experiments presented. Finally, we conclude the presentation with $\S 6$ where we summarise extensive numerical experiments aimed at testing the performance of the indicator as a basis for model adaptation.

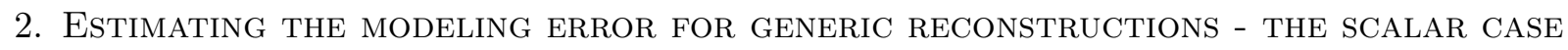

Our goal in this section is to show how the entropic structure can be used to obtain estimates for the difference between a (sufficiently regular) solution to (1.1) and the solution $\boldsymbol{v}_{h}$ of a numerical scheme which is a discretisation of (1.2) on part of the (space-time) domain and of (1.1) everywhere else. We will pay particular attention to the model adaptation error, i.e., the part of the error which is due to approximating not (1.1) but (1.2) on part of the domain. In this section we present the arguments in the scalar case, as in this case all arguments can be given in a tangible way, see $\S 2.2$.

2.1. Relative Entropy. Before treating the scalar case we will review the classical concept of entropy/entropy flux pair which will be used in this Section and in $\S 4$. We will show how it can be employed in the relative entropy stability framework. We will also show explicitly that the relative entropy in the NSF model satisfies the conditions which are necessary for our analysis. Throughout this exposition we will use $d$ to denote the spatial dimension of the problem and $n$ as the number of equations in the system.

Definition 2.1 (Entropy pair). We call a tuple $(\eta, \mathbf{q}) \in \mathrm{C}^{1}(U, \mathbb{R}) \times C^{1}\left(U, \mathbb{R}^{d}\right)$ an entropy pair to (1.1) provided the following compatibility conditions are satisfied:

$$
\mathrm{D} \eta \mathrm{D} \boldsymbol{f}_{\alpha}=\mathrm{D} q_{\alpha} \text { for } \alpha=1, \ldots, d
$$

and

$$
\partial_{x_{\alpha}}(\mathrm{D} \eta(\boldsymbol{y})): \boldsymbol{g}_{\alpha}(\boldsymbol{y}, \nabla \boldsymbol{y}) \geq 0 \quad \text { for any } \boldsymbol{y} \in \mathrm{C}^{1}\left(\mathbb{T}^{d}, U\right) \text { and } \alpha=1, \ldots, d
$$

where $\mathrm{D}$ denotes the Jacobian of functions defined on $U$ (the state space). We call an entropy pair strictly convex if $\eta$ is strictly convex.

Remark 2.2 (Entropy equality). Note that every solution $\boldsymbol{u} \in \mathrm{H}^{1}\left(\mathbb{T}^{d} \times(0, T), U\right)$ of (1.1) satisfies the additional companion balance law

$$
\partial_{t} \eta(\boldsymbol{u})+\sum_{\alpha} \partial_{x_{\alpha}}\left(q_{\alpha}(\boldsymbol{u})-\varepsilon \boldsymbol{g}_{\alpha}(\boldsymbol{u}, \nabla \boldsymbol{u}) \mathrm{D} \eta(\boldsymbol{u})\right)=-\varepsilon \sum_{\alpha} \boldsymbol{g}_{\alpha}(\boldsymbol{u}, \nabla \boldsymbol{u}) \partial_{x_{\alpha}} \mathrm{D} \eta(\boldsymbol{u})
$$

We refer to $\sum_{\alpha} \boldsymbol{g}_{\alpha}(\boldsymbol{u}, \nabla \boldsymbol{u}) \partial_{x_{\alpha}} \mathrm{D} \eta(\boldsymbol{u})$ as entropy dissipation.

Remark 2.3 (Entropic structure). We restrict our attention to systems (1.1) which are endowed with at least one strictly convex entropy pair. Such a convex entropy pair gives rise to a stability theory based on the relative entropy which we recall in Definition 2.5. We will make the additional assumption that 
$\eta \in \mathrm{C}^{3}(U, \mathbb{R})$. While this last assumption is not standard in relative entropy estimates, it does not exclude any important cases.

Remark 2.4 (Commutation property). Note that the existence of $q_{\alpha}$ means that for each $\alpha$ the vector field $\boldsymbol{u} \mapsto \mathrm{D} \eta(\boldsymbol{u}) \mathrm{D} \boldsymbol{f}_{\alpha}(\boldsymbol{u})$ has a potential and, thus, gives rise to the following commutation property:

$$
\left(\mathrm{D} \boldsymbol{f}_{\alpha}\right)^{\boldsymbol{\top}} \mathrm{D}^{2} \eta=\mathrm{D}^{2} \eta \mathrm{D} \boldsymbol{f}_{\alpha} \quad \text { for } \alpha=1, \ldots, d .
$$

Definition 2.5 (Relative entropy). Let (1.1) be endowed with an entropy pair $(\eta, \boldsymbol{q})$. Then the relative entropy and relative entropy flux between the states $\boldsymbol{u}, \boldsymbol{v} \in U$ are defined by

$$
\begin{aligned}
& \eta(\boldsymbol{u} \mid \boldsymbol{v}):=\eta(\boldsymbol{u})-\eta(\boldsymbol{v})-\mathrm{D} \eta(\boldsymbol{v})(\boldsymbol{u}-\boldsymbol{v}) \\
& \boldsymbol{q}(\boldsymbol{u} \mid \boldsymbol{v}):=\boldsymbol{q}(\boldsymbol{u})-\boldsymbol{q}(\boldsymbol{v})-\mathrm{D} \eta(\boldsymbol{v})(\boldsymbol{f}(\boldsymbol{u})-\boldsymbol{f}(\boldsymbol{v})) .
\end{aligned}
$$

Hypothesis 2.6 (Existence of reconstruction). In the remainder of this section we will assume existence of a reconstruction $\widehat{\boldsymbol{v}}$ of a numerical solution $\boldsymbol{v}_{h}$ which weakly solves

$$
\partial_{t} \widehat{\boldsymbol{v}}+\operatorname{div} \boldsymbol{f}(\widehat{\boldsymbol{v}})=\operatorname{div}(\widehat{\varepsilon} \boldsymbol{g}(\widehat{\boldsymbol{v}}, \nabla \widehat{\boldsymbol{v}}))+\mathscr{R}_{H}+\mathscr{R}_{P}
$$

with explicitly computable residuals $\mathscr{R}_{H}, \mathscr{R}_{P}$ and $\widehat{\varepsilon}: \mathbb{T}^{d} \times[0, T] \rightarrow[0, \varepsilon]$ being a function which is a consequence of the model adaptation procedure and determines in which part of the space-time domain which model is discretised. We assume that the residual can be split into a part denoted $\mathscr{R}_{H} \in \mathrm{L}_{2}\left(\mathbb{T}^{d} \times(0, T), \mathbb{R}^{n}\right)$ and a part proportional to $\widehat{\varepsilon}$, denoted $\mathscr{R}_{P}$, which is an element of $\mathrm{L}_{2}\left(0, T ; \mathrm{H}^{-1}\left(\mathbb{T}^{d}, \mathbb{R}^{n}\right)\right)$.

We also assume that an explicitly computable bound for $\left|\widehat{\boldsymbol{v}}-\boldsymbol{v}_{h}\right|$ is available.

Remark 2.7 (Model interpolation function). Our theory is applicable for arbitrary non-negative functions $\widehat{\varepsilon} \in L^{\infty}\left(\mathbb{T}^{d} \times(0, T)\right)$. In our numerical experiments, in $\S 6$, we use a function $\widehat{\varepsilon}$ only taking values in $\{0, \varepsilon\}$ and which is piecewise constant within computational cells and time-steps in order to avoid introducing cells in which we have an intermediate model. However, other choices are possible, and continuous choices for $\widehat{\varepsilon}$ will be explored in a later work.

Remark 2.8 (Reconstructions). We present some reconstructions in $\S 5$ and point out that different choices of reconstructions will lead to different behaviours of the residuals $\mathscr{R}_{H}, \mathscr{R}_{P}$ with respect to the mesh width $h$. However, our main interest in the work at hand is a rigorous estimation of the modelling error, and not the derivation of optimal order discretisation error estimates, which is a challenging task.

Throughout this paper we will make the following assumption on the exact solution.

Hypothesis 2.9 (Values in a compact set). We assume that we have a priori knowledge of a pair $(T, \mathfrak{O})$, where $T>0$ and $\mathfrak{O} \subset U$ is compact and convex, such that the exact solution $\boldsymbol{u}$ of (1.1) takes values in $\mathfrak{O}$ up to time $T$, i.e.,

$$
\boldsymbol{u}(\boldsymbol{x}, t) \in \mathfrak{O} \quad \forall(\boldsymbol{x}, t) \in \mathbb{T}^{d} \times[0, T] .
$$

2.2. A posteriori analysis of the scalar case. In the scalar setting, i.e., $n=1$, we restrict ourselves to the "complex" model being given by

$$
\partial_{t} u+\operatorname{div}(\boldsymbol{f}(u))=\operatorname{div}(\varepsilon \nabla u)
$$

and the simple model by

$$
\partial_{t} u+\operatorname{div}(\boldsymbol{f}(u))=0
$$

Therefore, our model adaptive algorithm can be viewed as a numerical discretisation of

$$
\partial_{t} u+\operatorname{div}(\boldsymbol{f}(u))=\operatorname{div}(\widehat{\varepsilon} \nabla u)
$$

with a space and time dependent function $\widehat{\varepsilon}$ taking values in $[0, \varepsilon]$. The spatial distribution of $\widehat{\varepsilon}$ determines which model is solved on which part of the domain. The reconstruction $\widehat{v}$ of the numerical solution is a Lipschitz continuous weak solution to the perturbed problem

$$
\partial_{t} \widehat{v}+\operatorname{div}(\boldsymbol{f}(\widehat{v}))=\operatorname{div}(\widehat{\varepsilon} \nabla \widehat{v})+\mathscr{R}_{H}+\mathscr{R}_{P},
$$

where $\widehat{\varepsilon}$ is a space dependent function with values in $[0, \varepsilon], \mathscr{R}_{H}$ is the "hyperbolic" part of the discretisation residual which is in $\mathrm{L}_{2}\left(\mathbb{T}^{d} \times(0, T), \mathbb{R}^{n}\right)$, and $\mathscr{R}_{P}$ is the "parabolic" part of the discretisation residual. Note that $\mathscr{R}_{P}$ is not in $\mathrm{L}_{2}\left(\mathbb{T}^{d} \times(0, T), \mathbb{R}^{n}\right)$ but in $\mathrm{L}_{2}\left(0, T ; \mathrm{H}^{-1}\left(\mathbb{T}^{d}, \mathbb{R}^{n}\right)\right)$ and that $\left\|\mathscr{R}_{P}\right\|_{\mathrm{L}_{2}\left(\mathrm{H}^{-1}\right)}$ is proportional to $\widehat{\varepsilon}$. See Hypothesis 2.6 for our general assumption and (5.16) for such a splitting in case of a specific reconstruction.

In what follows we will use the relative entropy stability framework to derive a bound for the difference between the solution $u$ of (2.6) and the reconstruction $\widehat{v}$ of the numerical solution. In the scalar case every 
strictly convex $\eta \in \mathrm{C}^{2}(U, \mathbb{R})$ is an entropy for (2.6), because to each such $\eta$ a consistent entropy flux may be defined by

$$
q_{\alpha}(u):=\int^{u} \eta^{\prime}(v) f_{\alpha}^{\prime}(v) \mathrm{d} v \text { for } \alpha=1, \ldots, d
$$

and the compatibility with the diffusive term boils down to

$$
-\left(\partial_{x_{\alpha}} y\right) \eta^{\prime \prime}(y)\left(\partial_{x_{\alpha}} y\right) \leq 0 \quad \text { for all } y \in \mathrm{C}^{1}(U, \mathbb{R}) \text { and } \alpha=1, \ldots, d
$$

which is satisfied as a consequence of the convexity of $\eta$.

We choose $\eta(u)=\frac{1}{2} u^{2}$ as this simplifies the subsequent calculations. In particular,

for all $u, v \in U$.

$$
\eta(u \mid v)=\frac{1}{2}(u-v)^{2}
$$

Remark 2.10 (Stability framework). Note that in the scalar case we might also use Kruzhkov's $\mathrm{L}_{1}$ stability framework [Kru70] instead of the relative entropy. However, the exposition at hand is supposed to serve as a blueprint for what we are going to do for systems in $\S 4$.

Remark 2.11 (Bounds on flux). Due to the regularity of $\boldsymbol{f}$ and the compactness of $\mathfrak{O}$ there exists a constant $0<C_{\overline{\boldsymbol{f}}}<\infty$ such that

$$
\left|f^{\prime \prime}(u)\right| \leq C_{\bar{f}} \quad \forall u \in \mathfrak{O}
$$

where $|\cdot|$ is the Euclidean norm for vectors. Note that $C_{\overline{\boldsymbol{f}}}$ can be explicitly computed from $\mathfrak{O}$ and $\boldsymbol{f}$.

The main result of this Section is then the following Theorem:

Theorem 2.12 (A posteriori modelling error control). Let $u \in \mathrm{H}^{1}\left(\mathbb{T}^{d} \times(0, T), \mathbb{R}\right)$ be a solution to (2.6) and let $\widehat{v} \in \mathrm{W}^{1, \infty}\left(\mathbb{T}^{d} \times(0, T), \mathbb{R}\right)$ solve $(2.9)$. Then, for almost all $t \in(0, T)$ the following bound for the difference between $u$ and $\widehat{v}$ holds:

$$
\begin{aligned}
\|u(\cdot, t)-\widehat{v}(\cdot, t)\|_{\mathrm{L}_{2}\left(\mathbb{T}^{d}\right)}^{2}+ & \int_{0}^{t} \varepsilon|u(\cdot, s)-\widehat{v}(\cdot, s)|_{\mathrm{H}^{1}\left(\mathbb{T}^{d}\right)}^{2} \mathrm{~d} s \\
& \leq\left(\|u(\cdot, 0)-\widehat{v}(\cdot, 0)\|_{\mathrm{L}_{2}\left(\mathbb{T}^{d}\right)}^{2}+\mathscr{E}_{M}+\mathscr{E}_{D}\right) \exp \left(\left(\|\nabla \widehat{v}\|_{\mathrm{L}_{\infty}\left(\mathbb{T}^{d} \times(0, t)\right)} C_{\overline{\boldsymbol{f}}}+1\right) t\right),
\end{aligned}
$$

with

$$
\begin{aligned}
& \mathscr{E}_{M}:=\left\|(\varepsilon-\widehat{\varepsilon})^{1 / 2} \nabla \widehat{v}\right\|_{L_{2}\left(\mathbb{T}^{d} \times(0, t)\right)}^{2}, \\
& \mathscr{E}_{D}:=\left\|\mathscr{R}_{H}\right\|_{\mathrm{L}_{2}\left(\mathbb{J}^{d} \times(0, t)\right)}^{2}+\frac{1}{\varepsilon}\left\|\mathscr{R}_{P}\right\|_{\mathrm{L}_{2}\left(0, t ; \mathrm{H}^{-1}\left(\mathbb{J}^{d}\right)\right)}^{2} .
\end{aligned}
$$

Remark 2.13 (Dependence of the estimator on $\varepsilon$.). We expect that the modelling residual part of the estimator in $(2.13), \mathscr{E}_{M}$, becomes small in large parts of the computational domain in case $\varepsilon$ is sufficiently small, even if $\widehat{\varepsilon}$ vanishes everywhere. This means that (1.2) is a reasonable approximation of (1.1). It should be noted that $\left\|\mathscr{R}_{P}\right\|_{\mathrm{L}_{2}\left(0, t ; \mathrm{H}^{-1}\left(\mathbb{T}^{d}\right)\right)} \sim \mathscr{O}(\varepsilon)$ (as $\widehat{\varepsilon}$ only takes the values 0 and $\varepsilon$ ), therefore we expect $\frac{1}{\varepsilon}\left\|\mathscr{R}_{P}\right\|_{\mathrm{L}_{2}\left(0, t ; \mathrm{H}^{-1}\left(\mathbb{T}^{d}\right)\right)}^{2} \sim \mathscr{O}(\varepsilon)$.

Proof of Theorem 2.12. Testing (2.6) and (2.9) with $(u-\widehat{v})$ and subtracting both equations we obtain

$$
\begin{aligned}
\int_{\mathbb{T}^{d}} \frac{1}{2} \partial_{t}\left((u-\widehat{v})^{2}\right)-\nabla \widehat{v}\left(\boldsymbol{f}(u)-\boldsymbol{f}(\widehat{v})-\boldsymbol{f}^{\prime}(\widehat{v})\right. & (u-\widehat{v}))+\varepsilon|\nabla(u-\widehat{v})|^{2} \\
= & \int_{\mathbb{T}^{d}}(\widehat{\varepsilon}-\varepsilon) \nabla \widehat{v} \cdot \nabla(u-\widehat{v})+\mathscr{R}_{H}(u-\widehat{v})+\mathscr{R}_{P}(u-\widehat{v}),
\end{aligned}
$$

where we have used that $\mathbb{T}^{d}$ has no boundary and

$$
\operatorname{div}(\boldsymbol{q}(u \mid \widehat{v}))-\nabla \widehat{v}\left(\boldsymbol{f}(u)-\boldsymbol{f}(\widehat{v})-\boldsymbol{f}^{\prime}(\widehat{v})(u-\widehat{v})\right)=\operatorname{div}(\boldsymbol{f}(u)-\boldsymbol{f}(\widehat{v}))(u-\widehat{v}) .
$$

Applying Young's inequality in (2.15) we obtain

$$
\begin{aligned}
& \mathrm{d}_{t}\left(\frac{1}{2}\|u-\widehat{v}\|_{\mathrm{L}_{2}\left(\mathbb{T}^{d}\right)}^{2}\right)+\varepsilon|(u-\widehat{v})|_{\mathrm{H}^{1}\left(\mathbb{T}^{d}\right)}^{2} \\
& \leq\left\|(\varepsilon-\widehat{\varepsilon})^{1 / 2} \nabla \widehat{v}\right\|_{\mathrm{L}_{2}\left(\mathbb{J}^{d}\right)}^{2}+\frac{\varepsilon}{4}|u-\widehat{v}|_{\mathrm{H}^{1}\left(\mathbb{T}^{d}\right)}^{2}+\left\|\mathscr{R}_{H}\right\|_{\mathrm{L}_{2}\left(\mathbb{T}^{d}\right)}^{2}+\|u-\widehat{v}\|_{\mathrm{L}_{2}\left(\mathbb{J}^{d}\right)}^{2} \\
& \quad+\frac{1}{\varepsilon}\left\|\mathscr{R}_{P}\right\|_{\mathrm{H}^{-1}\left(\mathbb{T}^{d}\right)}^{2}+\frac{\varepsilon}{4}|u-\widehat{v}|_{\mathrm{H}^{1}\left(\mathbb{J}^{d}\right)}^{2}+|\widehat{v}|_{\mathrm{W}^{1, \infty}\left(\mathbb{T}^{d}\right)} C_{\bar{f}}\|u-\widehat{v}\|_{\mathrm{L}_{2}\left(\mathbb{T}^{d}\right)}^{2} .
\end{aligned}
$$


Several terms in (2.17) cancel each other and we obtain

$$
\begin{aligned}
& \mathrm{d}_{t}\left(\frac{1}{2}\|u-\widehat{v}\|_{\mathrm{L}_{2}\left(\mathbb{T}^{d}\right)}^{2}\right)+\frac{\varepsilon}{2}|(u-\widehat{v})|_{\mathrm{H}^{1}\left(\mathbb{J}^{d}\right)}^{2} \\
& \leq\left\|(\varepsilon-\widehat{\varepsilon})^{1 / 2} \nabla \widehat{v}\right\|_{\mathrm{L}_{2}\left(\mathbb{T}^{d}\right)}^{2}+\left\|\mathscr{R}_{H}\right\|_{\mathrm{L}_{2}\left(\mathbb{T}^{d}\right)}^{2} \\
& \quad+\frac{1}{\varepsilon}\left\|\mathscr{R}_{P}\right\|_{\mathrm{H}^{-1}\left(\mathbb{T}^{d}\right)}^{2}+\left(|\widehat{v}|_{\mathrm{W}^{1, \infty}\left(\mathbb{T}^{d}\right)} C_{\bar{f}}+1\right)\|u-\widehat{v}\|_{\mathrm{L}_{2}\left(\mathbb{T}^{d}\right)}^{2} .
\end{aligned}
$$

Integrating (2.18) in time implies the assertion of the theorem.

\section{Some structural properties of isothermal Navier-Stokes and Navier-Stokes-Fourier EQUATIONS}

As pointed out before we are mainly interested in model adaptation between isothermal Navier-Stokes (INS) and isothermal Euler and between Navier-Stokes-Fourier (NSF) and Euler. Still, we will derive the a posteriori error estimates on an abstract level. These abstract arguments rely, in particular, on some structural properties which are satisfied by the systems mentioned above. In addition to existence of a convex entropy pair we need certain compatibility properties between the relative entropy and diffusive fluxes $\boldsymbol{g}$ as well as the parabolic part of the residual $\mathscr{R}_{P}$. The purpose of this section is to state those properties explicitly and to verify that they are satisfied for INS and NSF.

Let us begin by introducing the INS and NSF systems. Note that in this Section the notation differs from that in the rest of the paper so that the physical quantities under consideration are denoted as is standard in the fluid mechanics literature. The isothermal Navier-Stokes system (INS), where we replace the Navier-Stokes stress by $\nabla \mathbf{v}$ for simplicity, reads:

$$
\begin{aligned}
\partial_{t} \rho+\operatorname{div}(\rho \mathbf{v}) & =0 \\
\partial_{t}(\rho \mathbf{v})+\operatorname{div}(\rho \mathbf{v} \otimes \mathbf{v})+\nabla(p(\rho)) & =\operatorname{div}(\mu \nabla \mathbf{v})
\end{aligned}
$$

where $\rho$ denotes density, $\mathbf{v}$ denotes velocity and $p=p(\rho)$ is the pressure, given by a constitutive relation as a monotone function of density, and $\mu \geq 0$ is the viscosity parameter.

In this case the simple model are the isothermal Euler equations which are obtained from (3.1) by setting $\mu=0$. For these models the vector of conserved quantities is $\boldsymbol{u}=(\rho, \rho \mathbf{v})^{\mathbf{\top}}$ and the mathematical entropy is given by

$$
\eta(\rho, \rho \mathbf{v})=W(\rho)+\frac{|\rho \mathbf{v}|^{2}}{2 \rho},
$$

where Helmholtz energy $W$ and pressure $p$ are related by the Gibbs-Duhem relation

$$
p^{\prime}(\rho)=\rho W^{\prime \prime}(\rho) \text {. }
$$

The Navier-Stokes-Fourier (NSF) equations for an ideal gas, where we again replace the Navier-Stokes stress by $\nabla \mathbf{v}$, differ from the INS equations by also containing a conservation law for the energy:

$$
\begin{aligned}
\partial_{t} \rho+\operatorname{div}(\rho \mathbf{v}) & =0 \\
\partial_{t}(\rho \mathbf{v})+\operatorname{div}(\rho \mathbf{v} \otimes \mathbf{v})+\nabla(p(\rho, \epsilon)) & =\operatorname{div}(\mu \nabla \mathbf{v}) \\
\partial_{t} e+\operatorname{div}((e+p) \mathbf{v}) & =\operatorname{div}(\mu(\nabla \mathbf{v}) \cdot \mathbf{v}+\kappa \nabla T),
\end{aligned}
$$

where we understand $(\nabla \mathbf{v}) \cdot \mathbf{v}$ by $((\nabla \mathbf{v}) \cdot \mathbf{v})_{\alpha}=\sum_{\beta} v_{\beta} \partial_{x_{\alpha}} v_{\beta}$. The variables and parameters which appear in (3.2) and did not appear before are the temperature $T$, the specific inner energy $\epsilon$, and the heat conductivity $\kappa>0$. In an ideal gas it holds

$$
\begin{aligned}
& e=\rho \epsilon+\frac{1}{2} \rho|\mathbf{v}|^{2}, \\
& p=(\gamma-1) \rho \epsilon=\rho R T,
\end{aligned}
$$

where $R$ is the specific gas constant and $\gamma$ is the adiabatic coefficient. For air it holds $R=287$ and $\gamma=1.4$. In this case the simple model are the Euler equations which are obtained from (3.2) by setting $\mu, \kappa=0$. The vector of conserved quantities is $\boldsymbol{u}=(\rho, \rho \mathbf{v}, e)^{\mathbf{\top}}$ and the (mathematical) entropy is given by

$$
\eta(\boldsymbol{u})=-\rho \ln \left(\frac{p}{\rho^{\gamma}}\right) .
$$

We will impose in both cases that the state space $\mathfrak{O}$ enforces positive densities. 
Let us now state the compatibility conditions:

Definition 3.1 (Relative entropy compatible). We call a system (1.1) and a subspace $V \subset \mathbb{R}^{n}$ relative entropy compatible provided there exists a computable function $\mathscr{D}: U \times \mathbb{R}^{n \times d} \times U \times \mathbb{R}^{n \times d} \rightarrow[0, \infty)$ and a computable constant $k>0$ such that for all $\boldsymbol{w}, \widetilde{\boldsymbol{w}} \in \mathrm{W}^{1, \infty}\left(\mathbb{T}^{d}\right)$ taking values in $\mathfrak{O}$ and all $\mathscr{R} \in \mathrm{H}^{-1}\left(\mathbb{T}^{d}\right)$, taking values in $V$, the following holds

$$
\begin{aligned}
\sum_{\alpha}\left(\boldsymbol{g}_{\alpha}(\boldsymbol{w}, \nabla \boldsymbol{w})-\boldsymbol{g}_{\alpha}(\widetilde{\boldsymbol{w}}, \nabla \widetilde{\boldsymbol{w}})\right): \partial_{x_{\alpha}}(\mathrm{D} \eta(\boldsymbol{w}) & -\mathrm{D} \eta(\widetilde{\boldsymbol{w}})) \\
& \geq \frac{1}{k} \mathscr{D}(\boldsymbol{w}, \nabla \boldsymbol{w}, \widetilde{\boldsymbol{w}}, \nabla \widetilde{\boldsymbol{w}})-k\left(|\boldsymbol{w}|_{\mathrm{W}^{1, \infty}}^{2}+|\widetilde{\boldsymbol{w}}|_{\mathrm{W}^{1, \infty}}^{2}\right) \eta(\boldsymbol{w} \mid \widetilde{\boldsymbol{w}})
\end{aligned}
$$

and

$$
\begin{aligned}
& |\nabla(\mathrm{D} \eta(\boldsymbol{w})-\mathrm{D} \eta(\widetilde{\boldsymbol{w}})) \cdot \boldsymbol{g}(\widetilde{\boldsymbol{w}}, \nabla \widetilde{\boldsymbol{w}})| \\
& \quad \leq k^{2}\left(|\boldsymbol{w}|_{\mathrm{W}^{1, \infty}}^{2}+|\widetilde{\boldsymbol{w}}|_{\mathrm{W}^{1, \infty}}^{2}+1\right) \eta(\boldsymbol{w} \mid \widetilde{\boldsymbol{w}})+\frac{1}{2 k} \mathscr{D}(\boldsymbol{w}, \nabla \boldsymbol{w}, \widetilde{\boldsymbol{w}}, \nabla \widetilde{\boldsymbol{w}})+k^{2} \sum_{\alpha} \boldsymbol{g}_{\alpha}(\widetilde{\boldsymbol{w}}, \nabla \widetilde{\boldsymbol{w}}) \partial_{x_{\alpha}} \mathrm{D} \eta(\widetilde{\boldsymbol{w}})
\end{aligned}
$$

and

$$
\begin{aligned}
& \int_{\mathbb{T}^{d}} \mathscr{R}(\mathrm{D} \eta(\boldsymbol{w})-\mathrm{D} \eta(\widetilde{\boldsymbol{w}})) \\
& \quad \leq k\|\mathscr{R}\|_{\mathrm{H}^{-1}\left(\mathbb{T}^{d}\right)}\left(\left(|\boldsymbol{w}|_{\mathrm{W}^{1, \infty}}+|\widetilde{\boldsymbol{w}}|_{\mathrm{W}^{1, \infty}}+1\right)\left(\int_{\mathbb{T}^{d}} \eta(\boldsymbol{w} \mid \widetilde{\boldsymbol{w}})\right)^{\frac{1}{2}}+\left(\int_{\mathbb{T}^{d}} \mathscr{D}(\boldsymbol{w}, \nabla \boldsymbol{w}, \widetilde{\boldsymbol{w}}, \nabla \widetilde{\boldsymbol{w}})\right)^{\frac{1}{2}}\right) .
\end{aligned}
$$

The reason for introducing the space $V$ in (3.6) is that for any reasonable discretisation of the isothermal Navier-Stokes equations there is no parabolic residual in the mass conservation equation. The same is true for Navier-Stokes-Fourier.

We will now verify the relative entropy compatibility for the INS system.

Lemma 3.2. The isothermal Navier-Stokes equations (3.1) and the space $V=\{0\} \times \mathbb{R}^{d}$ are relative entropy compatible.

Proof. For the isothermal Navier-Stokes equations (3.1) the diffusive fluxes are given by

$$
\boldsymbol{g}_{\alpha}=\left(0, \partial_{x_{\alpha}} \mathbf{v}\right)^{\mathbf{\top}}
$$

so that in the inequalities in Definition 3.1 the derivative $\frac{\partial \eta}{\partial \rho}$ does not enter. We compute

$$
\frac{\partial \eta}{\partial(\rho \mathbf{v})}=\frac{\rho \mathbf{v}}{\rho}=\mathbf{v}
$$

Thus, in this case entropy dissipation is given by

$$
\mu \sum_{\alpha} \boldsymbol{g}_{\alpha}(\boldsymbol{w}, \nabla \boldsymbol{w}) \partial_{x_{\alpha}} \mathrm{D} \eta(\boldsymbol{w})=\mu|\nabla \mathbf{v}|^{2},
$$

where $|\cdot|$ denotes the Frobenius norm and $\boldsymbol{w}=(\rho, \rho \mathbf{v})^{\boldsymbol{\top}}$. With $\widetilde{\boldsymbol{w}}=(\widetilde{\rho}, \widetilde{\rho} \widetilde{\mathbf{v}})^{\boldsymbol{\top}}$ we define

$$
\mathscr{D}(\boldsymbol{w}, \nabla \boldsymbol{w}, \widetilde{\boldsymbol{w}}, \nabla \widetilde{\boldsymbol{w}}):=|\nabla \mathbf{v}-\nabla \widetilde{\mathbf{v}}|^{2} .
$$

Let us now verify that there exists a constant $k$ such that the inequalities from Definition 3.1 are valid. Making use of the identities (3.7) and (3.8) we obtain

$$
(\boldsymbol{g}(\boldsymbol{w}, \nabla \boldsymbol{w})-\boldsymbol{g}(\widetilde{\boldsymbol{w}}, \nabla \widetilde{\boldsymbol{w}})): \nabla(\mathrm{D} \eta(\boldsymbol{w})-\mathrm{D} \eta(\widetilde{\boldsymbol{w}}))=(\nabla \mathbf{v}-\nabla \widetilde{\mathbf{v}}):(\nabla \mathbf{v}-\nabla \widetilde{\mathbf{v}})=\mathscr{D}(\boldsymbol{w}, \nabla \boldsymbol{w}, \widetilde{\boldsymbol{w}}, \nabla \widetilde{\boldsymbol{w}})
$$

so that (3.4) is satisfied for any $k \geq 1$. Now, again using (3.7) and (3.8), we find for any $k \geq 1$

$$
\begin{aligned}
& |\nabla(\mathrm{D} \eta(\boldsymbol{w})-\mathrm{D} \eta(\widetilde{\boldsymbol{w}})) \cdot \boldsymbol{g}(\widetilde{\boldsymbol{w}}, \nabla \widetilde{\boldsymbol{w}})|=|\nabla(\mathbf{v}-\widetilde{\mathbf{v}}): \nabla \widetilde{\mathbf{v}}| \\
& \leq \frac{1}{2 k}|\nabla(\mathbf{v}-\widetilde{\mathbf{v}})|^{2}+k|\nabla \widetilde{\mathbf{v}}|^{2}=\frac{1}{2 k} \mathscr{D}(\boldsymbol{w}, \nabla \boldsymbol{w}, \widetilde{\boldsymbol{w}}, \nabla \widetilde{\boldsymbol{w}})+k \sum_{\alpha} \boldsymbol{g}_{\alpha}(\widetilde{\boldsymbol{w}}, \nabla \widetilde{\boldsymbol{w}}) \partial_{x_{\alpha}} \mathrm{D} \eta(\widetilde{\boldsymbol{w}}),
\end{aligned}
$$

i.e., (3.5) is satisfied for any $k \geq 1$.

For any $\mathscr{R} \in \mathrm{H}^{-1}\left(\mathbb{T}^{d}\right)$, with values in $\{0\} \times \mathbb{R}^{d}$, we have

$$
\begin{aligned}
\int_{\mathbb{T}^{d}} \mathscr{R}(\mathrm{D} \eta(\boldsymbol{w})-\mathrm{D} \eta(\widetilde{\boldsymbol{w}})) \leq\|\mathscr{R}\|_{\mathrm{H}^{-1}}\|\mathbf{v}-\widetilde{\mathbf{v}}\|_{\mathrm{H}^{1}} \\
\leq\|\mathscr{R}\|_{\mathrm{H}^{-1}}\left(k\|\boldsymbol{w}-\widetilde{\boldsymbol{w}}\|_{\mathrm{L}_{2}}+\left(\int_{\mathbb{T}^{d}} \mathscr{D}(\boldsymbol{w}, \nabla \boldsymbol{w}, \widetilde{\boldsymbol{w}}, \nabla \widetilde{\boldsymbol{w}})\right)^{\frac{1}{2}}\right)
\end{aligned}
$$


which shows that (3.6) is satisfied with a constant $k$ depending only on $\mathfrak{O}$.

Next, we show relative entropy compatibility for the NSF system. This system contains two parameters $\mu, \kappa$ scaling different dissipative mechanisms. We will identify $\mu$ with the small parameter in (1.1) and keep the ratio $\frac{\kappa}{\mu}$, which we will treat as a constant, of order 1 .

Lemma 3.3. The Navier-Stokes-Fourier equations for an ideal gas (3.2) and the space $V=\{0\} \times \mathbb{R}^{d} \times \mathbb{R}$ are relative entropy compatible.

Proof. For NSF the diffusive flux is $\boldsymbol{g}_{\alpha}=\left(0, \partial_{x_{\alpha}} \mathbf{v}, \mathbf{v} \cdot \partial_{x_{\alpha}} \mathbf{v}+\frac{\kappa}{\mu} \partial_{x_{\alpha}} T\right)^{\boldsymbol{\top}}$. Therefore, the derivative $\frac{\partial \eta}{\partial \rho}$ does not enter the inequalities in Definition 3.1 and it is sufficient to compute

$$
\frac{\partial \eta}{\partial(\rho \mathbf{v})}=\frac{\gamma-1}{R} \frac{\mathbf{v}}{T} ; \quad \frac{\partial \eta}{\partial e}=-\frac{\gamma-1}{R} \frac{1}{T}
$$

for understanding relations in Definition 3.1. From equation (3.13) we may compute entropy dissipation:

$$
\mu \sum_{\alpha} \boldsymbol{g}_{\alpha}(\boldsymbol{u}, \nabla \boldsymbol{u}) \partial_{x_{\alpha}} \mathrm{D} \eta(\boldsymbol{u})=\frac{\gamma-1}{R} \frac{\mu}{T}|\nabla \mathbf{v}|^{2}+\kappa \frac{\gamma-1}{R} \frac{|\nabla T|^{2}}{T^{2}}
$$

and we define

$$
\mathscr{D}(\boldsymbol{w}, \nabla \boldsymbol{w}, \widetilde{\boldsymbol{w}}, \nabla \widetilde{\boldsymbol{w}}):=\frac{1}{\widetilde{T}}|\nabla \mathbf{v}-\nabla \widetilde{\mathbf{v}}|^{2}+\frac{\kappa}{\mu} \frac{|\nabla T-\nabla \widetilde{T}|^{2}}{\widetilde{T}^{2}} .
$$

Note that $\mathscr{D}(\boldsymbol{w}, \nabla \boldsymbol{w}, \widetilde{\boldsymbol{w}}, \nabla \widetilde{\boldsymbol{w}})$ is not symmetric in $\boldsymbol{w}$ and $\widetilde{\boldsymbol{w}}$. Let us now determine a constant $k$ such that the conditions from Definition 3.1 are valid. By inserting the definitions of $\boldsymbol{g}$ and $\eta$ into the left hand side of (3.4) we obtain

$$
\begin{aligned}
(\boldsymbol{g}(\boldsymbol{w}, \nabla \boldsymbol{w})-\boldsymbol{g}(\widetilde{\boldsymbol{w}}, \nabla \widetilde{\boldsymbol{w}})): \nabla(\mathrm{D} \eta(\boldsymbol{w})-\mathrm{D} \eta(\widetilde{\boldsymbol{w}})) & \\
=\frac{\gamma-1}{R}\left[\nabla\left(\frac{\mathbf{v}}{T}-\frac{\widetilde{\mathbf{v}}}{T}\right): \nabla(\mathbf{v}-\widetilde{\mathbf{v}})-\nabla\left(\frac{1}{T}-\frac{1}{\widetilde{T}}\right)(\nabla \mathbf{v} \cdot \mathbf{v}-\nabla \widetilde{\mathbf{v}} \cdot \widetilde{\mathbf{v}})\right. & \left.\quad-\frac{\kappa}{\mu} \nabla\left(\frac{1}{T}-\frac{1}{\widetilde{T}}\right) \nabla(T-\widetilde{T})\right] .
\end{aligned}
$$

After a lengthy but straightforward computation we arrive at

$$
\begin{aligned}
& \frac{R}{\gamma-1}(\boldsymbol{g}(\boldsymbol{w}, \nabla \boldsymbol{w})-\boldsymbol{g}(\widetilde{\boldsymbol{w}}, \nabla \widetilde{\boldsymbol{w}})): \nabla(\mathrm{D} \eta(\boldsymbol{w})-\mathrm{D} \eta(\widetilde{\boldsymbol{w}})) \\
& \quad=\frac{1}{\widetilde{T}}|\nabla(\mathbf{v}-\widetilde{\mathbf{v}})|^{2}-\frac{\nabla \widetilde{T}}{\widetilde{T}^{2}}(\nabla \mathbf{v}-\nabla \widetilde{\mathbf{v}})(\mathbf{v}-\widetilde{\mathbf{v}}) \\
& +\left(\frac{1}{T}-\frac{1}{\widetilde{T}}\right) \nabla \mathbf{v}: \nabla(\mathbf{v}-\widetilde{\mathbf{v}})-\left(\frac{1}{T^{2}}-\frac{1}{\widetilde{T}^{2}}\right) \nabla T \nabla \widetilde{\mathbf{v}}(\mathbf{v}-\widetilde{\mathbf{v}}) \\
& \quad+\frac{\nabla(T-\widetilde{T})}{\widetilde{T}^{2}} \nabla \widetilde{\mathbf{v}}(\mathbf{v}-\widetilde{\mathbf{v}})+\frac{\kappa}{\mu} \frac{|\nabla(T-\widetilde{T})|^{2}}{\widetilde{T}^{2}}+\frac{\kappa}{\mu}\left(\frac{1}{T^{2}}-\frac{1}{\widetilde{T}^{2}}\right) \nabla T \nabla(T-\widetilde{T}) .
\end{aligned}
$$

Note that the two summands of $\mathscr{D}$ both appear on the right hand side of (3.17). Applying Young's inequality to the other terms on the right hand side of (3.17) shows that (3.4) is true for some $k$ only depending on $\mathfrak{O}$.

By inserting the particular forms of $\boldsymbol{g}$ and $\eta$ for NSF into the left hand side of (3.5) we obtain

$$
\begin{aligned}
& \frac{R}{\gamma-1}|\nabla(\mathrm{D} \eta(\boldsymbol{w})-\mathrm{D} \eta(\widetilde{\boldsymbol{w}})) \cdot \boldsymbol{g}(\widetilde{\boldsymbol{w}}, \nabla \widetilde{\boldsymbol{w}})| \\
& \quad \leq \nabla\left(\frac{\mathbf{v}}{T}-\frac{\widetilde{\mathbf{v}}}{T}\right) \nabla \widetilde{\mathbf{v}}-\nabla\left(\frac{1}{T}-\frac{1}{\widetilde{T}}\right) \nabla \widetilde{\mathbf{v}} \cdot \widetilde{\mathbf{v}}-\frac{\kappa}{\mu} \nabla\left(\frac{1}{T}-\frac{1}{\widetilde{T}}\right) \nabla \widetilde{T}
\end{aligned}
$$

We may rewrite this as

$$
\begin{aligned}
\frac{R}{\gamma-1} \mid \nabla(\mathrm{D} \eta(\boldsymbol{w})- & \mathrm{D} \eta(\widetilde{\boldsymbol{w}})) \cdot \boldsymbol{g}(\widetilde{\boldsymbol{w}}, \nabla \widetilde{\boldsymbol{w}}) \mid \\
\leq & \frac{\nabla \mathbf{v}-\nabla \widetilde{\mathbf{v}}}{T}: \nabla \widetilde{\mathbf{v}}-\frac{\nabla T}{T^{2}} \nabla \widetilde{\mathbf{v}}(\mathbf{v}-\widetilde{\mathbf{v}})+\left(\frac{1}{T}-\frac{1}{\widetilde{T}}\right) \nabla \widetilde{\mathbf{v}}: \nabla \widetilde{\mathbf{v}} \\
& \quad+\frac{\kappa}{\mu} \frac{1}{\widetilde{T}^{2}} \nabla(T-\widetilde{T}) \cdot \nabla \widetilde{T}+\frac{\kappa}{\mu}\left(\frac{1}{T^{2}}-\frac{1}{\widetilde{T}^{2}}\right) \nabla T \cdot \nabla \widetilde{T}
\end{aligned}
$$


Using Young's inequality we may infer from (3.19) that (3.5) is true for some $k$ only depending on $\mathfrak{O}$.

Finally, for any $\mathscr{R}$ taking values in $\{0\} \times \mathbb{R}^{d+1}$ we have

$$
\begin{aligned}
& \frac{R}{\gamma-1} \int_{\mathbb{T}^{d}} \mathscr{R}(\mathrm{D} \eta(\boldsymbol{w})-\mathrm{D} \eta(\widetilde{\boldsymbol{w}})) \leq\|\mathscr{R}\|_{\mathrm{H}^{-1}}\left(\left\|\frac{\mathbf{v}}{T}-\frac{\widetilde{\mathbf{v}}}{\widetilde{T}}\right\|_{\mathrm{H}^{1}}+\left\|\frac{1}{T}-\frac{1}{\widetilde{T}}\right\|_{\mathrm{H}^{1}}\right) \\
& \leq\|\mathscr{R}\|_{\mathrm{H}^{-1}}\left(C\left(|\boldsymbol{w}|_{\mathrm{W}^{1, \infty}}+|\widetilde{\boldsymbol{w}}|_{\mathrm{W}^{1, \infty}}+1\right)\|\boldsymbol{w}-\widetilde{\boldsymbol{w}}\|_{\mathrm{L}_{2}}+\left(\int_{\mathbb{T}^{d}} \mathscr{D}(\boldsymbol{w}, \nabla \boldsymbol{w}, \widetilde{\boldsymbol{w}}, \nabla \widetilde{\boldsymbol{w}})\right)^{\frac{1}{2}}\right)
\end{aligned}
$$

which shows that (3.6) is satisfied with a constant $k$ depending only on $\mathfrak{O}$.

\section{Estimating the MODELing ERROR FOR GENERIC RECONSTRUCTIONS - THE SYSTEMS CASE}

In this section we set up a more abstract framework allowing for the analysis of systems of equations. This framework relies of the relative entropy compatibility, Definition 3.1. For clarity of exposition we do not explicitly track the constants, rather denote a generic constant $C$ which may depend on $\mathfrak{O}, \boldsymbol{f}, \boldsymbol{g}, \eta$ but is independent of mesh size, solution and $\varepsilon$. Robustness of the estimates in $\varepsilon$ is of particular importance since we are particularly interested in the case of small $\varepsilon$.

As in $\S 2$ we assume Hypothesis 2.9 which gives rise to the following bounds:

Remark 4.1 (Bounds on flux and entropy). Due to the regularity of $\boldsymbol{f}$ and $\eta$, and the compactness of $\mathfrak{O}$ there are constants $0<C_{\bar{f}}<\infty$ and $0<C_{\underline{\eta}}<C_{\bar{\eta}}<\infty$ such that

$$
\left|\boldsymbol{v}^{\boldsymbol{\top}} \mathrm{D}^{2} \boldsymbol{f}(\boldsymbol{u}) \boldsymbol{v}\right| \leq C_{\overline{\boldsymbol{f}}}|\boldsymbol{v}|^{2}, \quad C_{\underline{\eta}}|\boldsymbol{v}|^{2} \leq \boldsymbol{v}^{\boldsymbol{\top}} \mathrm{D}^{2} \eta(\boldsymbol{u}) \boldsymbol{v} \leq C_{\bar{\eta}}|\boldsymbol{v}|^{2} \quad \forall \boldsymbol{v} \in \mathbb{R}^{n}, \boldsymbol{u} \in \mathfrak{O},
$$

and

$$
\left|\mathrm{D}^{3} \eta(\boldsymbol{u})\right| \leq C_{\bar{\eta}} \quad \forall \boldsymbol{u} \in \mathfrak{O},
$$

where $|\cdot|$ is the Euclidean norm for vectors in (4.1) and a Frobenius norm for 3-tensors in (4.2). Note that $C_{\overline{\boldsymbol{f}}}, C_{\underline{\eta}}$ and $C_{\bar{\eta}}$ can be explicitly computed from $\mathfrak{O}, \boldsymbol{f}$ and $\eta$.

Now we are in position to state the main result of this section

Theorem 4.2 (A posteriori modelling error control). Let $\boldsymbol{u} \in \mathrm{W}^{1, \infty}\left(\mathbb{T}^{d} \times(0, T), \mathbb{R}^{n}\right)$ be a weak solution to (1.1). Let $\widehat{\boldsymbol{v}} \in \mathrm{W}^{1, \infty}\left(\mathbb{T}^{d} \times(0, T), \mathbb{R}^{n}\right)$ weakly solve $(2.5)$, with $\mathscr{R}_{P}$ taking values in some subspace $V \subset \mathbb{R}^{n}$. Let (1.1) be endowed with a strictly convex entropy pair, and let (1.1) and $V$ be relative entropy compatible. Let $\boldsymbol{u}$ and $\widehat{\boldsymbol{v}}$ only take values in $\mathfrak{O}$. Then, the following a posteriori error estimate holds:

$$
\begin{aligned}
\|\boldsymbol{u}(\cdot, t)-\widehat{\boldsymbol{v}}(\cdot, t)\|_{\mathrm{L}_{2}\left(\mathbb{T}^{d}\right)}^{2}+ & \int_{\mathbb{T}^{d} \times(0, t)} \frac{\varepsilon}{4 k} \mathscr{D}(\boldsymbol{u}, \nabla \boldsymbol{u}, \widehat{\boldsymbol{v}}, \nabla \widehat{\boldsymbol{v}}) \\
& \leq C\left(\|\boldsymbol{u}(\cdot, 0)-\widehat{\boldsymbol{v}}(\cdot, 0)\|_{\mathrm{L}_{2}\left(\mathbb{T}^{d}\right)}^{2}+\mathscr{E}_{D}+\mathscr{E}_{M}\right) \\
& \times \exp \left(C\left(\|\boldsymbol{u}\|_{\mathrm{W}^{1, \infty}}^{2}+\| \widehat{\boldsymbol{v}}_{\mathrm{W}^{1, \infty}}^{2}+1\right) t\right)
\end{aligned}
$$

with $C, k$ being constants depending on $(\mathfrak{O}, \boldsymbol{f}, \boldsymbol{g}, \eta)$ and

$$
\begin{aligned}
\mathscr{E}_{M} & :=\|\widehat{\varepsilon} \boldsymbol{g}(\widehat{\boldsymbol{v}}, \nabla \widehat{\boldsymbol{v}})\|_{\mathrm{L}_{2}\left(\mathbb{T}^{d} \times(0, t)\right)}^{2}+\int_{\mathbb{T}^{d} \times(0, t)}(\varepsilon-\widehat{\varepsilon}) k^{2} \sum_{\alpha} \boldsymbol{g}_{\alpha}(\widehat{\boldsymbol{v}}, \nabla \widehat{\boldsymbol{v}}) \partial_{x_{\alpha}} \mathrm{D} \eta(\widehat{\boldsymbol{v}}), \\
\mathscr{E}_{D} & :=\frac{k^{2}}{\varepsilon}\left\|\mathscr{R}_{P}\right\|_{\mathrm{L}_{2}\left(0, t ; \mathrm{H}^{-1}\left(\mathbb{T}^{d}\right)\right)}^{2}+\left\|\mathscr{R}_{H}\right\|_{\mathrm{L}_{2}\left(\mathbb{T}^{d} \times(0, t)\right)}^{2} .
\end{aligned}
$$

Remark 4.3 (Energy dissipation degeneracy). It should be noted that the estimate in Theorem 4.2 contains certain assumptions, i.e., $\boldsymbol{u} \in \mathrm{W}^{1, \infty}$, which are not verifiable in an a posteriori fashion. In particular, we assume more regularity than can be expected for solutions of systems of the form (1.1); see [FNS11, FJN12] for existence results for systems of this type. However, the weak solutions defined in those references are not unique and only weak-strong uniqueness results are available, cf. [FJN12]. Thus, convergent a posteriori error estimators can only be expected in case the problem (1.1) has a more regular solution than is guaranteed analytically. Note that the corresponding term, i.e., $\|\boldsymbol{u}\|_{\mathrm{W}^{1, \infty}}$ does not appear in the scalar case and is a consequence of the dissipation only being present in momentum and energy conservation laws but not in the mass conservation equation. This leads to a form of degeneracy of the energy dissipation governing the underlying system.

In addition, the presence of $\|\boldsymbol{u}\|_{\mathrm{W}^{1, \infty}}$ on the right hand side of (4.3) makes it impossible to compute this quantity a posteriori without knowledge of the exact solution. Practically, we expect $\|\boldsymbol{u}\|_{\mathrm{W}^{1, \infty}}$ to be smaller than $\|\widehat{\boldsymbol{v}}\|_{\mathrm{W}^{1, \infty}}$ since $\widehat{\boldsymbol{v}}$ results from a system with less dissipation, but to the best of our knowledge there is 
no guarantee that this is the case. For practical computations we believe that $\mathscr{E}_{D}, \mathscr{E}_{M}$ are good indicators for discretisation and modelling errors, respectively.

Remark 4.4 (Structure of the estimator). Note that the first factor in the right hand side of (4.3) consists of three parts. The first part is the error in the discretisation and reconstruction of the initial data. The second part $\mathscr{E}_{D}$ is due to the residuals caused by the discretisation error. The third part $\mathscr{E}_{M}$ consists of residuals caused by the model approximation error.

Remark 4.5 (Structure of modelling error residual). Recall that we are interested in the case of $\varepsilon$ being (very) small. In this case the term

$$
\int_{\mathbb{T}^{d} \times(0, t)}(\varepsilon-\widehat{\varepsilon}) k^{2} \sum_{\alpha} \boldsymbol{g}_{\alpha}(\widehat{\boldsymbol{v}}, \nabla \widehat{\boldsymbol{v}}) \partial_{x_{\alpha}} \mathrm{D} \eta(\widehat{\boldsymbol{v}})
$$

is the dominating part in the modelling error residual $\mathscr{E}_{M}$ and, thus, letting $\widehat{\varepsilon}$ be $\varepsilon$ in larger parts of the domain will usually reduce the modelling error residual.

Proof of Theorem 4.2. We test (1.1) by $\mathrm{D} \eta(\boldsymbol{u})-\mathrm{D} \eta(\widehat{\boldsymbol{v}})$ and $(2.5)$ by $\mathrm{D}^{2} \eta(\boldsymbol{u})(\boldsymbol{u}-\widehat{\boldsymbol{v}})$ and subtract both equations. By rearranging terms and using (2.3) we obtain

$$
\int_{\mathbb{T}^{d}} \partial_{t} \eta(\boldsymbol{u} \mid \widehat{\boldsymbol{v}})+\sum_{\alpha} \partial_{x_{\alpha}} \boldsymbol{q}_{\alpha}(\boldsymbol{u} \mid \widehat{\boldsymbol{v}})+\varepsilon(\boldsymbol{g}(\boldsymbol{u}, \nabla \boldsymbol{u})-\boldsymbol{g}(\widehat{\boldsymbol{v}}, \nabla \widehat{\boldsymbol{v}})): \nabla(\mathrm{D} \eta(\boldsymbol{u})-\mathrm{D} \eta(\widehat{\boldsymbol{v}}))=E_{1}+E_{2}+E_{3},
$$

with

$$
\begin{aligned}
& E_{1}:=\int_{\mathbb{T}^{d}} \widehat{\varepsilon} \boldsymbol{g}(\widehat{\boldsymbol{v}}, \nabla \widehat{\boldsymbol{v}}): \nabla\left(\mathrm{D}^{2} \eta(\widehat{\boldsymbol{v}})(\boldsymbol{u}-\widehat{\boldsymbol{v}})\right)-\varepsilon \boldsymbol{g}(\widehat{\boldsymbol{v}}, \nabla \widehat{\boldsymbol{v}}): \nabla(\mathrm{D} \eta(\boldsymbol{u})-\mathrm{D} \eta(\widehat{\boldsymbol{v}})), \\
& E_{2}:=-\int_{\mathbb{J}^{d}} \sum_{\alpha} \partial_{x_{\alpha}} \widehat{\boldsymbol{v}} \mathrm{D}^{2} \eta(\widehat{\boldsymbol{v}})\left(\boldsymbol{f}_{\alpha}(\boldsymbol{u})-\boldsymbol{f}_{\alpha}(\widehat{\boldsymbol{v}})-\mathrm{D} \boldsymbol{f}_{\alpha}(\widehat{\boldsymbol{v}})(\boldsymbol{u}-\widehat{\boldsymbol{v}})\right), \\
& E_{3}:=\int_{\mathbb{T}^{d}}\left(\mathscr{R}_{H}+\mathscr{R}_{P}\right) \mathrm{D}^{2} \eta(\widehat{\boldsymbol{v}})(\boldsymbol{u}-\widehat{\boldsymbol{v}}) .
\end{aligned}
$$

As $\mathbb{T}^{d}$ does not have a boundary and because of (3.4) we obtain

$$
\int_{\mathbb{J}^{d}} \partial_{t} \eta(\boldsymbol{u} \mid \widehat{\boldsymbol{v}})+\frac{\varepsilon}{k} \mathscr{D}(\boldsymbol{u}, \nabla \boldsymbol{u}, \widehat{\boldsymbol{v}}, \nabla \widehat{\boldsymbol{v}}) \leq E_{1}+E_{2}+E_{3}+\varepsilon k\left(|\boldsymbol{u}|_{\mathrm{W}^{1, \infty}}^{2}+|\widehat{\boldsymbol{v}}|_{\mathrm{W}^{1, \infty}}^{2}\right) \int_{\mathbb{T}^{d}} \eta(\boldsymbol{u} \mid \widehat{\boldsymbol{v}}) .
$$

We are now going to derive estimates for the terms $E_{1}, E_{2}, E_{3}$ on the right hand side of (4.7). We may rewrite $E_{1}$ as

$$
E_{1}=E_{11}+E_{12}
$$

with

$$
\begin{aligned}
\left|E_{11}\right|:=\mid \int_{\mathbb{J}^{d}} \widehat{\varepsilon} \boldsymbol{g}(\widehat{\boldsymbol{v}}, \nabla \widehat{\boldsymbol{v}}): \nabla\left(\mathrm{D}^{2} \eta(\widehat{\boldsymbol{v}})(\boldsymbol{u}-\widehat{\boldsymbol{v}})\right. & -\mathrm{D} \eta(\boldsymbol{u})+\mathrm{D} \eta(\widehat{\boldsymbol{v}})) \mid \\
& \leq\|\widehat{\varepsilon} \boldsymbol{g}(\widehat{\boldsymbol{v}}, \nabla \widehat{\boldsymbol{v}})\|_{\mathrm{L}_{2}}^{2}+C_{\bar{\eta}}\left(\|\boldsymbol{u}\|_{\mathrm{W}^{1, \infty}}^{2}+\|\widehat{\boldsymbol{v}}\|_{\mathrm{W}^{1, \infty}}^{2}\right)\|\boldsymbol{u}-\widehat{\boldsymbol{v}}\|_{\mathrm{L}_{2}}^{2},
\end{aligned}
$$

where we used (4.2) and

$$
E_{12}:=-\int_{\mathbb{T}^{d}}(\varepsilon-\widehat{\varepsilon}) \boldsymbol{g}(\widehat{\boldsymbol{v}}, \nabla \widehat{\boldsymbol{v}}): \nabla(\mathrm{D} \eta(\boldsymbol{u})-\mathrm{D} \eta(\widehat{\boldsymbol{v}}))
$$

Using (3.5) we find

$$
\begin{aligned}
\left|E_{12}\right| \leq \varepsilon k^{2}\left(|\boldsymbol{u}|_{\mathrm{W}^{1, \infty}}^{2}+|\widehat{\boldsymbol{v}}|_{\mathrm{W}^{1, \infty}}^{2}+1\right) \eta(\boldsymbol{u} \mid \widehat{\boldsymbol{v}}) & \\
& +\frac{\varepsilon}{2 k} \mathscr{D}(\boldsymbol{u}, \nabla \boldsymbol{u}, \widehat{\boldsymbol{v}}, \nabla \widehat{\boldsymbol{v}})+(\varepsilon-\widehat{\varepsilon}) k^{2} \sum_{\alpha} \boldsymbol{g}_{\alpha}(\widehat{\boldsymbol{v}}, \nabla \widehat{\boldsymbol{v}}) \partial_{x_{\alpha}} \mathrm{D} \eta(\widehat{\boldsymbol{v}}) .
\end{aligned}
$$

Concerning $E_{2}$ we note

$$
\left|E_{2}\right| \leq C_{\bar{\eta}} C_{\overline{\boldsymbol{f}}}|\widehat{\boldsymbol{v}}|_{\mathrm{W}^{1, \infty}}\|\boldsymbol{u}-\widehat{\boldsymbol{v}}\|_{\mathrm{L}_{2}}^{2}
$$

We decompose $E_{3}$ into two terms

$$
E_{3}=\int_{\mathbb{T}^{d}} \mathscr{R}_{H} \mathrm{D}^{2} \eta(\widehat{\boldsymbol{v}})(\boldsymbol{u}-\widehat{\boldsymbol{v}})+\mathscr{R}_{P} \mathrm{D}^{2} \eta(\widehat{\boldsymbol{v}})(\boldsymbol{u}-\widehat{\boldsymbol{v}})=: E_{31}+E_{32} .
$$

We have

$$
\left|E_{31}\right| \leq\left\|\mathscr{R}_{H}\right\|_{\mathrm{L}_{2}}^{2}+C_{10}^{2}\|\boldsymbol{u}-\widehat{\boldsymbol{v}}\|_{\mathrm{L}_{2}}^{2}
$$


We rewrite $E_{32}$ as

$$
E_{32}=\int_{\mathbb{T}^{d}} \mathscr{R}_{P}\left(-\mathrm{D} \eta(\boldsymbol{u})+\mathrm{D} \eta(\widehat{\boldsymbol{v}})+\mathrm{D}^{2} \eta(\widehat{\boldsymbol{v}})(\boldsymbol{u}-\widehat{\boldsymbol{v}})\right)+\mathscr{R}_{P}(\mathrm{D} \eta(\boldsymbol{u})-\mathrm{D} \eta(\widehat{\boldsymbol{v}}))
$$

such that we get the following estimate

$$
\begin{aligned}
\left|E_{32}\right| \leq\left\|\mathscr{R}_{P}\right\|_{\mathrm{H}^{-1}\left(\mathbb{T}^{d}\right)}\left\|\mathrm{D} \eta(\boldsymbol{u})-\mathrm{D} \eta(\widehat{\boldsymbol{v}})-\mathrm{D}^{2} \eta(\widehat{\boldsymbol{v}})(\boldsymbol{u}-\widehat{\boldsymbol{v}})\right\|_{\mathrm{H}^{1}\left(\mathbb{J}^{d}\right)} \\
\quad+k\left\|\mathscr{R}_{P}\right\|_{\mathrm{H}^{-1}\left(\mathbb{T}^{d}\right)}\left(\left(|\boldsymbol{u}|_{\mathrm{W}^{1, \infty}}+|\widehat{\boldsymbol{v}}|_{\mathrm{W}^{1, \infty}}+1\right)\|\boldsymbol{u}-\widehat{\boldsymbol{v}}\|_{\mathrm{L}_{2}\left(\mathbb{J}^{d}\right)}+\left(\int_{\mathbb{T}^{d}} \mathscr{D}(\boldsymbol{u}, \nabla \boldsymbol{u}, \widehat{\boldsymbol{v}}, \nabla \widehat{\boldsymbol{v}})\right)^{\frac{1}{2}}\right)
\end{aligned}
$$

due to (3.6). We have

$$
\left\|\mathrm{D} \eta(\boldsymbol{u})-\mathrm{D} \eta(\widehat{\boldsymbol{v}})-\mathrm{D}^{2} \eta(\widehat{\boldsymbol{v}})(\boldsymbol{u}-\widehat{\boldsymbol{v}})\right\|_{\mathrm{H}^{1}} \leq C_{\bar{\eta}}\left(\|\boldsymbol{u}\|_{\mathrm{W}^{1, \infty}}+\|\widehat{\boldsymbol{v}}\|_{\mathrm{W}^{1, \infty}}\right)\|\boldsymbol{u}-\widehat{\boldsymbol{v}}\|_{\mathrm{L}_{2}},
$$

such that (4.16) becomes

$$
\left|E_{32}\right| \leq \frac{k^{2}}{\varepsilon}\left\|\mathscr{R}_{P}\right\|_{\mathrm{H}^{-1}}^{2}+2 \varepsilon C_{\bar{\eta}}^{2}\left(\|\boldsymbol{u}\|_{\mathrm{W}^{1, \infty}}^{2}+\|\widehat{\boldsymbol{v}}\|_{\mathrm{W}^{1, \infty}}^{2}+1\right)\|\boldsymbol{u}-\widehat{\boldsymbol{v}}\|_{\mathrm{L}_{2}}^{2}+\frac{\varepsilon}{4 k} \int_{\mathbb{T}^{d}} \mathscr{D}(\boldsymbol{u}, \nabla \boldsymbol{u}, \widehat{\boldsymbol{v}}, \nabla \widehat{\boldsymbol{v}}) .
$$

Combining (4.14) and (4.18) we obtain

$$
\begin{aligned}
\left|E_{3}\right| \leq \frac{k^{2}}{\varepsilon}\left\|\mathscr{R}_{P}\right\|_{\mathrm{H}^{-1}}^{2}+2 C_{\bar{\eta}}^{2}\left(\varepsilon\|\boldsymbol{u}\|_{\mathrm{W}^{1, \infty}}^{2}+\varepsilon\|\widehat{\boldsymbol{v}}\|_{\mathrm{W}^{1, \infty}}^{2}+2\right)\|\boldsymbol{u}-\widehat{\boldsymbol{v}}\|_{\mathrm{L}_{2}}^{2} & \\
& +\left\|\mathscr{R}_{H}\right\|_{\mathrm{L}_{2}}^{2}+\frac{\varepsilon}{4 k} \int_{\mathbb{J}^{d}} \mathscr{D}(\boldsymbol{u}, \nabla \boldsymbol{u}, \widehat{\boldsymbol{v}}, \nabla \widehat{\boldsymbol{v}}) .
\end{aligned}
$$

Upon inserting (4.9), (4.11), (4.12) and (4.19) into (4.7) we obtain for $\varepsilon<1$

$$
\begin{aligned}
\int_{\mathbb{T}^{d}} \partial_{t} \eta(\boldsymbol{u} \mid \widehat{\boldsymbol{v}})+\frac{\varepsilon}{4 k} \mathscr{D}(\boldsymbol{u}, \nabla \boldsymbol{u}, \widehat{\boldsymbol{v}}, \nabla \widehat{\boldsymbol{v}}) & \\
\leq\|\widehat{\varepsilon} \boldsymbol{g}(\widehat{\boldsymbol{v}}, \nabla \widehat{\boldsymbol{v}})\|_{\mathrm{L}_{2}}^{2}+ & C\left(|\boldsymbol{u}|_{\mathrm{W}^{1, \infty}}^{2}+|\widehat{\boldsymbol{v}}|_{\mathrm{W}^{1, \infty}}^{2}+1\right) \int_{\mathbb{T}^{d}} \eta(\boldsymbol{u} \mid \widehat{\boldsymbol{v}}) \\
& +(\varepsilon-\widehat{\varepsilon}) k^{2} \sum_{\alpha} \boldsymbol{g}_{\alpha}(\widehat{\boldsymbol{v}}, \nabla \widehat{\boldsymbol{v}}) \partial_{x_{\alpha}} \mathrm{D} \eta(\widehat{\boldsymbol{v}})+\frac{k^{2}}{\varepsilon}\left\|\mathscr{R}_{P}\right\|_{\mathrm{H}^{-1}}^{2}+\left\|\mathscr{R}_{H}\right\|_{\mathrm{L}_{2}}^{2}
\end{aligned}
$$

where we have used that $\|\boldsymbol{u}-\widehat{\boldsymbol{v}}\|_{\mathrm{L}_{2}}^{2}$ is bounded in terms of the relative entropy. Using Gronwall's Lemma we obtain

$$
\begin{aligned}
&\|\boldsymbol{u}(\cdot, t)-\widehat{\boldsymbol{v}}(\cdot, t)\|_{\mathrm{L}_{2}\left(\mathbb{T}^{d}\right)}^{2}+ \frac{\varepsilon}{4 k} \int_{\mathbb{T}^{d} \times(0, t)} \mathscr{D}(\boldsymbol{u}, \nabla \boldsymbol{u}, \widehat{\boldsymbol{v}}, \nabla \widehat{\boldsymbol{v}}) \mathrm{d} s \\
& \leq C_{\bar{\eta}}\left(\|\boldsymbol{u}(\cdot, 0)-\widehat{\boldsymbol{v}}(\cdot, 0)\|_{\mathrm{L}_{2}\left(\mathbb{T}^{d}\right)}^{2}+\mathscr{E}_{D}+\mathscr{E}_{M}\right) \\
& \quad \times \exp \left(C\left(|\boldsymbol{u}|_{\mathrm{W}^{1, \infty}}^{2}+\mid \widehat{\boldsymbol{v}}_{\mathrm{W}^{1, \infty}}^{2}+1\right) t\right)
\end{aligned}
$$

with $\mathscr{E}_{D}, \mathscr{E}_{M}$ defined in (4.4).

\section{Reconstructions}

In $\S 2$ and $\S 4$ we have assumed existence of reconstructions of numerical solutions whose residuals are computable, see Hypothesis 2.6. We have also assumed a certain regularity of these reconstructions. In this Section we will describe one way to obtain such reconstructions for semi-(spatially)-discrete dG schemes.

In previous works reconstructions for $\mathrm{dG}$ schemes have been mainly used for deriving a posteriori bounds of discretisation errors [DG15, GMP15, GHM14, c.f.] for hyperbolic problems. In these works the main idea is to compare the numerical solution $\boldsymbol{v}_{h}$ and the exact solution $\boldsymbol{u}$ not directly, but to introduce an intermediate quantity, the reconstruction $\widehat{\boldsymbol{v}}$ of the numerical solution. This reconstruction must have two crucial properties:

- Explicit a posteriori bounds for the difference $\left\|\widehat{\boldsymbol{v}}-\boldsymbol{v}_{h}\right\|_{\mathscr{X}}$ for some appropriate $\mathscr{X}$ need to be available and,

- The reconstruction $\widehat{\boldsymbol{v}}$ needs to be globally smooth enough to apply the appropriate stability theory of the underlying PDE. 
These two properties allow the derivation of an a posteriori bound for the difference $\left\|\boldsymbol{u}-\boldsymbol{v}_{h}\right\|_{\mathscr{X}}$.

In the sequel we will provide a methodology for the explicit computation of $\widehat{\boldsymbol{v}}$ only from the numerical solution $\boldsymbol{v}_{h}$. This means trivially that the difference $\left\|\widehat{\boldsymbol{v}}-\boldsymbol{v}_{h}\right\|_{\mathscr{X}}$ can be controlled explicitly.

From $\S 2$ and $\S 4$ the stability theory we advocate is that of relative entropy and we have extended the classical approach such that not only discretisation but also modelling errors are accounted for. Note also that for our results from $\S 2$ and $\S 4$ to be applicable we require $\widehat{\boldsymbol{v}} \in \mathrm{W}^{1, \infty}\left(\mathbb{T}^{d} \times[0, T]\right)$.

In this Section we describe how to obtain reconstructions $\widehat{\boldsymbol{v}}$ of numerical solutions $\boldsymbol{v}_{h}$ which are obtained by solving (1.1) on part of the space-time domain and (1.2) on the rest of the space-time domain. For brevity we will focus on numerical solutions obtained by semi-(spatially)-discrete dG schemes, which are a frequently used tool for the numerical simulation of models of the forms (1.1) and (1.2) alike. We will view $\boldsymbol{v}_{h}$ as a discretisation of the "intermediate" problem

$$
\partial_{t} \boldsymbol{v}+\operatorname{div} \boldsymbol{f}(\boldsymbol{v})=\operatorname{div}(\widehat{\varepsilon} \boldsymbol{g}(\boldsymbol{v}, \nabla \boldsymbol{v}))
$$

where $\widehat{\varepsilon}$ is the model adaptation function, which will be chosen as part of the numerical method.

Remark 5.1 (Alternative types of reconstruction). If (1.1) was a parabolic problem, this would be a quite strong argument in favour of using elliptic reconstruction, see [MN03], but this would make the residuals scale with $\frac{1}{\varepsilon}$. Recall that we are interested in the case of $\varepsilon$ being small. As important examples, e.g., the Navier-Stokes-Fourier equations, are not parabolic we will describe a reconstruction approach here which was developed for semi-discrete dG schemes for hyperbolic problems in one space dimension in [GMP15]. An extension to fully discrete methods can be found in [DG15].

Note that we state reconstructions in this paper to keep it self contained and to describe how we proceed in our numerical experiments in $\S 6$. It is, however, beyond the scope of this work to derive optimal reconstructions for (1.1), (1.2). For all of these problems the derivation of optimal reconstructions of the numerical solution is a problem in its own right. Note that in this framework optimality of a reconstruction means that the error estimator, which is obtained based on this reconstruction, is of the same order as the (true) error of the numerical scheme.

We will first outline the reconstructions for (1.2) in one space dimension, proposed in [GMP15], and investigate in which sense they lead to reconstructions of numerical solutions to (1.1) or (5.1). Afterwards we describe how the reconstruction approach can be extended to dG methods on Cartesian meshes in two space dimensions. We choose Cartesian meshes because they lend themselves to an extension of the approach from [GMP15]. We are not able to show the optimality of $\mathscr{R}_{H}$ in this case, though. Finding suitable (optimal) reconstructions for non-linear hyperbolic systems on unstructured meshes is the topic of ongoing research.

5.1. A reconstruction approach for $\mathbf{d G}$ approximations of hyperbolic conservation laws. In this section we recall a reconstruction approach for semi-(spatially)-discrete $d G$ schemes for systems of hyperbolic conservation laws (1.2) complemented with initial data $\boldsymbol{u}(\cdot, 0)=\boldsymbol{u}_{0} \in L^{\infty}(\mathbb{T})$. We consider the one dimensional case. An extension to fully discrete schemes can be found in [DG15]. Let $\mathscr{T}$ be a set of open intervals such that

$$
\bigcup_{S \in \mathscr{T}} \bar{S}=\mathbb{T} \text { (the } 1 \mathrm{~d} \text { torus), and for all } S_{1}, S_{2} \in \mathscr{T} \text { it holds } S_{1}=S_{2} \text { or } S_{1} \cap S_{2}=\emptyset \text {. }
$$

By $\mathscr{E}$ we denote the set of interval boundaries.

The space of piecewise polynomial functions of degree $q \in \mathbb{N}$ is defined by

$$
\mathbb{V}_{q}:=\left\{\boldsymbol{w}: \mathbb{T} \rightarrow \mathbb{R}^{n}:\left.\boldsymbol{w}\right|_{S} \in \mathbb{P}_{q}\left(S, \mathbb{R}^{n}\right) \forall S \in \mathscr{T}\right\},
$$

where $\mathbb{P}_{q}\left(S, \mathbb{R}^{n}\right)$ denotes the space of polynomials of degree $\leq q$ on $S$ with values in $\mathbb{R}^{n}$.

For defining our scheme we also need jump and average operators which require the definition of a broken Sobolev space:

Definition 5.2 (Broken Sobolev space). The broken Sobolev space $\mathrm{H}^{1}\left(\mathscr{T}, \mathbb{R}^{n}\right)$ is defined by

$$
\mathrm{H}^{1}\left(\mathscr{T}, \mathbb{R}^{n}\right):=\left\{\boldsymbol{w}: \mathbb{T} \rightarrow \mathbb{R}^{n}:\left.\boldsymbol{w}\right|_{S} \in \mathrm{H}^{1}\left(S, \mathbb{R}^{n}\right) \forall S \in \mathscr{T}\right\} .
$$

Definition 5.3 (Traces, jumps and averages). For any $\boldsymbol{w} \in \mathrm{H}^{1}\left(\mathscr{T}, \mathbb{R}^{n}\right)$ we define

- $\boldsymbol{w}^{ \pm}: \mathscr{E} \rightarrow \mathbb{R}^{n}$ by $\boldsymbol{w}^{ \pm}(\cdot):=\lim _{s \backslash 0} \boldsymbol{w}(\cdot \pm s)$

- $\left\{\{\boldsymbol{w}\}: \mathscr{E} \rightarrow \mathbb{R}^{n}\right.$ by $\left\{\{\boldsymbol{w}\}=\frac{\boldsymbol{w}^{-}+\boldsymbol{w}^{+}}{2}\right.$,

- $\llbracket \boldsymbol{w} \rrbracket: \mathscr{E} \rightarrow \mathbb{R}^{n}$ by $\llbracket \boldsymbol{w} \rrbracket=\boldsymbol{w}^{-}-\boldsymbol{w}^{+}$.

Now we are in position to state the numerical schemes under consideration: 
Definition 5.4 (Numerical scheme for (1.2)). The numerical scheme is to seek $\boldsymbol{v}_{h} \in \mathrm{C}^{1}\left((0, T), \mathbb{V}_{q}\right)$ such that:

$$
\begin{aligned}
& \boldsymbol{v}_{h}(0)=\mathscr{P}_{q}\left[\boldsymbol{u}_{0}\right] \\
& \int_{\mathscr{T}} \partial_{t} \boldsymbol{v}_{h} \cdot \boldsymbol{\phi}-\boldsymbol{f}\left(\boldsymbol{v}_{h}\right) \cdot \partial_{x} \boldsymbol{\phi}+\int_{\mathscr{E}} \boldsymbol{F}\left(\boldsymbol{v}_{h}^{-}, \boldsymbol{v}_{h}^{+}\right) \llbracket \boldsymbol{\phi} \rrbracket=0 \quad \text { for all } \boldsymbol{\phi} \in \mathbb{V}_{q},
\end{aligned}
$$

where $\int_{\mathscr{T}}$ is an abbreviation for $\sum_{S \in \mathscr{T}} \int_{S}, \mathscr{P}_{q}$ denotes $\mathrm{L}_{2}$-orthogonal projection into $\mathbb{V}_{q}$, and $\boldsymbol{F}: U \times U \rightarrow \mathbb{R}^{n}$ is a numerical flux function. We impose that the numerical flux function satisfies the following condition: There exist $L>0$ and $\boldsymbol{w}: U \times U \rightarrow U$ such that

$$
\boldsymbol{F}(\boldsymbol{u}, \boldsymbol{v})=\boldsymbol{f}(\boldsymbol{w}(\boldsymbol{u}, \boldsymbol{v})) \quad \text { for all } \boldsymbol{u}, \boldsymbol{v} \in U,
$$

and

$$
|\boldsymbol{w}(\boldsymbol{u}, \boldsymbol{v})-\boldsymbol{u}|+|\boldsymbol{w}(\boldsymbol{u}, \boldsymbol{v})-\boldsymbol{v}| \leq L|\boldsymbol{u}-\boldsymbol{v}| \quad \text { for all } \boldsymbol{u}, \boldsymbol{v} \in \mathfrak{O} .
$$

Remark 5.5 (Conditions on the flux). Note that conditions (5.6) and (5.7) imply the consistency and local Lipschitz continuity conditions usually imposed on numerical fluxes in the convergence analysis of $\mathrm{dG}$ approximations of hyperbolic conservation laws. The conditions do not make the flux monotone nor do they ensure stability of (5.5). They do, however, ensure that the right hand side of (5.5) is Lipschitz continuous and, therefore, (5.5) has unique solutions for small times. Obviously, practical interest is restricted to numerical fluxes leading to reasonable stability properties of (5.5) at least as long as the exact solution to (1.2) is Lipschitz continuous. Fluxes of Richtmyer and Lax-Wendroff type lead to stable numerical schemes (as long as the exact solution is smooth) and satisfy a relaxed version of conditions (5.6) and (5.7). It was shown in [DG15] that these relaxed conditions (see [DG15, Rem. 3.6]) are sufficient for obtaining optimal a posteriori error estimates.

Let us now return to the main purpose of this section: the definition of a reconstruction operator. In addition, we present a reconstruction of the numerical flux which will be used for splitting the residual into a parabolic and a hyperbolic part, in $§ 5.2$. They are based on information from the numerical scheme:

Definition 5.6 (Reconstructions). For each $t \in[0, T]$ we define the flux reconstruction $\widehat{\boldsymbol{f}}(\cdot, t) \in \mathbb{V}_{q+1}$ through

$$
\begin{aligned}
\int_{\mathscr{T}} \partial_{x} \widehat{\boldsymbol{f}}(\cdot, t) \cdot \boldsymbol{\phi} & =-\int_{\mathscr{T}} \boldsymbol{f}\left(\boldsymbol{v}_{h}(\cdot, t)\right) \cdot \partial_{x} \boldsymbol{\phi}+\int_{\mathscr{E}} \boldsymbol{F}\left(\boldsymbol{v}_{h}^{-}(\cdot, t), \boldsymbol{v}_{h}^{+}(\cdot, t)\right) \llbracket \boldsymbol{\phi} \rrbracket \quad \text { for all } \boldsymbol{\phi} \in \mathbb{V}_{q} \\
\hat{\boldsymbol{f}}^{+}(\cdot, t) & =\boldsymbol{F}\left(\boldsymbol{v}_{h}^{-}(\cdot, t), \boldsymbol{v}_{h}^{+}(\cdot, t)\right) \quad \text { on } \mathscr{E} .
\end{aligned}
$$

For each $t \in[0, T]$ we define the reconstruction $\widehat{\boldsymbol{v}}(\cdot, t) \in \mathbb{V}_{q+1}$ through

$$
\begin{aligned}
\int_{\mathbb{T}} \widehat{\boldsymbol{v}}(\cdot, t) \cdot \boldsymbol{\psi} & =\int_{\mathbb{T}} \boldsymbol{v}_{h}(\cdot, t) \cdot \boldsymbol{\psi} \text { for all } \boldsymbol{\psi} \in \mathbb{V}_{q-1} \\
\widehat{\boldsymbol{v}}^{ \pm}(\cdot, t) & =\boldsymbol{w}\left(\boldsymbol{v}_{h}^{-}(\cdot, t), \boldsymbol{v}_{h}^{+}(\cdot, t)\right) \quad \text { on } \mathscr{E} .
\end{aligned}
$$

Remark 5.7 (Properties of reconstruction). It was shown in [GMP15] that these reconstructions are welldefined, explicitly and locally computable and Lipschitz continuous in space. Due to the Lipschitz continuity of $\boldsymbol{w}$ they are also Lipschitz continuous in time. Recall from $\S 2$ and $\S 4$ that the Lipschitz continuity of $\widehat{\boldsymbol{v}}$ in space was crucial for our arguments.

Due to the Lipschitz continuity of $\widehat{\boldsymbol{v}}$ the definition of the discretisation residual satisfies

$$
\mathscr{R}:=\partial_{t} \widehat{\boldsymbol{v}}+\partial_{x} \boldsymbol{f}(\widehat{\boldsymbol{v}}) \in \mathrm{L}_{\infty} .
$$

At this point the reader might ask why we have defined $\widehat{f}$ as it is not present in (5.10) and is not needed for computing the residual $\mathscr{R}$ either. We will use $\widehat{f}$ in $\S 5.2$ to split the residual into a parabolic and a hyperbolic part. As a preparation to this end let us note that upon combing (5.5) and (5.8) we obtain

$$
\partial_{t} \boldsymbol{v}_{h}+\partial_{x} \widehat{\boldsymbol{f}}=0
$$

pointwise almost everywhere. Thus, we may split the residual as follows:

$$
\mathscr{R}=\partial_{t}\left(\widehat{\boldsymbol{v}}-\boldsymbol{v}_{h}\right)+\partial_{x}(\boldsymbol{f}(\widehat{\boldsymbol{v}})-\widehat{\boldsymbol{f}}) .
$$

This splitting was used in [GMP15] to argue that the residual is of optimal order. 
5.2. A reconstruction approach for $\mathbf{d G}$ approximations of hyperbolic/parabolic problems. We will describe in this Section how the reconstruction methodology described above can be used in case of $\mathrm{dG}$ semi- (spatial) discretisations of (5.1) in one space dimension following the local dG methodology.

Definition 5.8 (Discrete gradients). By $\partial_{x}^{-}, \partial_{x}^{+}: \mathrm{H}^{1}\left(\mathscr{T}, \mathbb{R}^{m}\right) \rightarrow \mathbb{V}_{q}$ we denote discrete gradient operators defined through

$$
\int_{\mathbb{T}} \partial_{x}^{ \pm} \boldsymbol{y} \cdot \boldsymbol{\phi}=-\int_{\mathscr{T}} \boldsymbol{y} \cdot \partial_{x} \boldsymbol{\phi}+\int_{\mathscr{E}} \boldsymbol{y}^{ \pm} \llbracket \phi \rrbracket \quad \text { for all } \boldsymbol{y}, \boldsymbol{\phi} \in \mathbb{V}_{q} .
$$

Lemma 5.9 (Discrete integration by parts). The operators $\partial_{x}^{ \pm}$satisfy the following duality property: For any $\phi, \psi \in \mathbb{V}_{q}$ it holds

$$
\int_{\mathbb{T}} \phi \partial_{x}^{-} \psi=-\int_{\mathbb{T}} \psi \partial_{x}^{+} \phi
$$

The proof of Lemma 5.9 can be found in [DPE12]. Rewriting (5.1) as

$$
\begin{aligned}
\boldsymbol{s} & =\partial_{x} \boldsymbol{v} \\
\partial_{t} \boldsymbol{v}+\partial_{x} \boldsymbol{f}(\boldsymbol{v}) & =\partial_{x}(\widehat{\varepsilon} \boldsymbol{g}(\boldsymbol{v}, \boldsymbol{s}))
\end{aligned}
$$

motivates the following semi-discrete numerical scheme:

Definition 5.10 (Numerical scheme). The numerical solution $\left(\boldsymbol{v}_{h}, \boldsymbol{s}_{h}\right) \in\left[\mathrm{C}^{1}\left((0, T), \mathbb{V}_{q}\right)\right]^{2}$ is given as the solution to

$$
\begin{aligned}
\boldsymbol{v}_{h}(0) & =\mathscr{P}_{q}\left[\boldsymbol{u}_{0}\right] \\
\boldsymbol{s}_{h} & =\partial_{x}^{-} \boldsymbol{v}_{h} \\
\int_{\mathscr{T}} \partial_{t} \boldsymbol{v}_{h} \cdot \boldsymbol{\phi}-\boldsymbol{f}\left(\boldsymbol{v}_{h}\right) \cdot \partial_{x} \boldsymbol{\phi}+\widehat{\varepsilon} \boldsymbol{g}\left(\boldsymbol{v}_{h}, \boldsymbol{s}_{h}\right) \partial_{x}^{-} \boldsymbol{\phi}+\int_{\mathscr{E}} \boldsymbol{F}\left(\boldsymbol{v}_{h}^{-}, \boldsymbol{v}_{h}^{+}\right) \llbracket \phi \rrbracket & =0 \quad \text { for all } \boldsymbol{\phi} \in \mathbb{V}_{q} .
\end{aligned}
$$

Defining $\widehat{\boldsymbol{f}}$ as in (5.8) allows us to rewrite $(5.13)_{3}$, using Lemma 5.9, as

$$
\partial_{t} \boldsymbol{v}_{h}+\partial_{x} \widehat{\boldsymbol{f}}-\partial_{x}^{+} \mathscr{P}_{q}\left[\widehat{\varepsilon} \boldsymbol{g}\left(\boldsymbol{v}_{h}, \partial_{x}^{-} \boldsymbol{v}_{h}\right)\right]=0 .
$$

Due to the arguments given in $\S 5.1$ the reconstruction $\widehat{\boldsymbol{v}}$ is an element of $\mathrm{W}^{1, \infty}\left(\mathbb{T} \times(0, T), \mathbb{R}^{n}\right)$ such that the following residual makes sense in $\mathrm{L}_{2}\left(0, T ; \mathrm{H}^{-1}(\mathbb{T})\right)$ :

$$
\mathscr{R}:=\partial_{t} \widehat{\boldsymbol{v}}+\partial_{x} \boldsymbol{f}(\widehat{\boldsymbol{v}})-\partial_{x}\left(\widehat{\varepsilon} \boldsymbol{g}\left(\widehat{\boldsymbol{v}}, \partial_{x} \widehat{\boldsymbol{v}}\right)\right) .
$$

Using (5.14) we may rewrite the residual as

$$
\mathscr{R}=\underbrace{\partial_{t}\left(\widehat{\boldsymbol{v}}-\boldsymbol{v}_{h}\right)+\partial_{x}(\boldsymbol{f}(\widehat{\boldsymbol{v}})-\widehat{\boldsymbol{f}})}_{=: \mathscr{R}_{H}}+\underbrace{\partial_{x}^{+} \mathscr{P}_{q}\left[\widehat{\varepsilon} \boldsymbol{g}\left(\boldsymbol{v}_{h}, \partial_{x}^{-} \boldsymbol{v}_{h}\right)\right]-\partial_{x}\left(\widehat{\varepsilon} \boldsymbol{g}\left(\widehat{\boldsymbol{v}}, \partial_{x} \widehat{\boldsymbol{v}}\right)\right)}_{=: \mathscr{R}_{P}},
$$

i.e., we have a decomposition of the residual as assumed in previous Sections, see (2.5) in particular.

5.3. Extension of the reconstruction to 2 space dimensions. In this section we present an extension of the reconstruction approach described before to semi-(spatially)-discrete dG schemes for systems of hyperbolic conservation laws (1.2) complemented with initial data $\boldsymbol{v}(\cdot, 0)=\boldsymbol{v}_{0} \in L^{\infty}\left(\mathbb{T}^{2}\right)$ using Cartesian meshes in two space dimensions. The extension to Cartesian meshes in more than two dimensions is straightforward.

We consider a system of hyperbolic conservation laws in two space dimensions

$$
\partial_{t} \boldsymbol{v}+\partial_{x_{1}} \boldsymbol{f}_{1}(\boldsymbol{v})+\partial_{x_{2}} \boldsymbol{f}_{2}(\boldsymbol{v})=0
$$

where $\boldsymbol{f}_{1,2} \in \mathrm{C}^{2}\left(U, \mathbb{R}^{n}\right)$.

We discretise $\mathbb{T}^{2}$ using partitions

$$
-1=x_{0}<x_{1}<\cdots<x_{N}=1, \quad-1=y_{0}<y_{1}<\cdots<y_{M}=1 .
$$

We consider a Cartesian mesh $\mathscr{T}$ such that each element satisfies $K=\left[x_{i}, x_{i+1}\right] \times\left[y_{j}, y_{j+1}\right]$ for some $(i, j) \in\{0, \ldots, N-1\} \times\{0, \ldots, M-1\}$. For any $p, q \in \mathbb{N}$ and $K \in \mathscr{T}$ let

$$
\mathbb{P}_{q} \otimes \mathbb{P}_{p}(K):=\mathbb{P}_{q}\left(\left[x_{i}, x_{i+1}\right]\right) \otimes \mathbb{P}_{p}\left(\left[y_{j}, y_{j+1}\right]\right) .
$$

By $\mathbb{V}_{p, q}$ we denote the space of trial and test functions, i.e.,

$$
\mathbb{V}_{p, q}:=\left\{\Phi: \mathbb{T}^{2} \rightarrow \mathbb{R}^{m}:\left.\Phi\right|_{K} \in\left(\mathbb{P}_{p} \otimes \mathbb{P}_{q}(K)\right)^{m} \forall K \in \mathscr{T}\right\} .
$$

Note that our dG space has a tensorial structure on each element. 
As before $\mathscr{E}$ denotes the set of all edges, which can be decomposed into the sets of horizontal and vertical edges $\mathscr{E}^{h}, \mathscr{E}^{v}$, respectively. Let us define the following jump operators: For $\Phi \in \mathrm{H}^{1}\left(\mathscr{T}, \mathbb{R}^{n}\right)$ we define

$$
\begin{aligned}
& \llbracket \Phi \rrbracket^{h}: \mathscr{E}^{v} \rightarrow \mathbb{R}^{n} \quad \llbracket \Phi \rrbracket^{h}(\cdot):=\lim _{s \searrow 0} \Phi\left(\cdot-s \boldsymbol{e}_{1}\right)-\lim _{s \searrow 0} \Phi\left(\cdot+s \boldsymbol{e}_{1}\right) \\
& \llbracket \Phi \rrbracket^{v}: \mathscr{E} h \rightarrow \mathbb{R}^{n}, \quad \llbracket \Phi \rrbracket^{v}(\cdot):=\lim _{s \searrow 0} \Phi\left(\cdot-s \boldsymbol{e}_{2}\right)-\lim _{s \searrow 0} \Phi\left(\cdot+s \boldsymbol{e}_{2}\right) .
\end{aligned}
$$

Let $\boldsymbol{F}_{1,2}$ be numerical flux functions which satisfy conditions (5.6) and (5.7) with functions $\boldsymbol{w}_{1}, \boldsymbol{w}_{2}$ : $U \times U \rightarrow U$, i.e.,

$$
\boldsymbol{F}_{i}(\boldsymbol{u}, \boldsymbol{v})=\boldsymbol{f}_{i}\left(\boldsymbol{w}_{i}(\boldsymbol{u}, \boldsymbol{v})\right) \quad \text { for all } \boldsymbol{u}, \boldsymbol{v} \in U \text { and } i=1,2 .
$$

Then, we consider semi-(spatially)-discrete discontinuous Galerkin schemes given as follows: Search for $\boldsymbol{v}_{h} \in \mathrm{C}^{1}\left([0, \infty), \mathbb{V}_{q, q}\right)$ satisfying

$$
\begin{aligned}
\int_{\mathscr{T}} \partial_{t} \boldsymbol{v}_{h} \Phi-\boldsymbol{f}_{1}(\boldsymbol{v}) \partial_{x_{1}} \Phi-\boldsymbol{f}_{2}(\boldsymbol{v}) \partial_{x_{2}} \Phi & \\
& +\int_{\mathscr{E} v} \boldsymbol{F}_{1}\left(\boldsymbol{v}_{h}^{-}, \boldsymbol{v}_{h}^{+}\right) \llbracket \Phi \rrbracket^{h}+\int_{\mathscr{E} h} \boldsymbol{F}_{2}\left(\boldsymbol{v}_{h}^{-}, \boldsymbol{v}_{h}^{+}\right) \llbracket \Phi \rrbracket^{v}=0 \quad \forall \Phi \in \mathbb{V}_{q, q} .
\end{aligned}
$$

While we have avoided choosing particular bases of our dG spaces we will do so now as we believe that it makes the presentation of our reconstruction approach more concise. We choose so-called nodal basis functions consisting of Lagrange polynomials, see [HW08], and as we use a Cartesian mesh we may use tensorproducts of one-dimensional Lagrange polynomials to this end. We associate the Lagrange polynomials with Gauss points, as in this way the nodal basis functions form an orthogonal basis of our dG space $\mathbb{V}_{q, q}$, due to the exactness properties of Gauss quadrature, see $\left[\mathrm{HGA}^{+} 12\right.$, e.g.]. We will introduce some notation now: Let $\left\{\xi_{0}, \ldots, \xi_{q}\right\}$ denote the Gauss points on $[-1,1]$. For any element $K=\left[x_{i}, x_{i+1}\right] \times\left[y_{j}, y_{j+1}\right] \in \mathscr{T}$ let $\left\{\xi_{0}^{K, 1}, \ldots, \xi_{q}^{K, 1}\right\}$ and $\left\{\xi_{0}^{K, 2}, \ldots, \xi_{q}^{K, 2}\right\}$ denote their image under the linear bijections $[-1,1] \rightarrow\left[x_{i}, x_{i+1}\right]$ and $[-1,1] \rightarrow\left[y_{j}, y_{j+1}\right]$. For $i=1,2$ we denote by $l_{j}^{K, i}$ the Lagrange polynomial satisfying $l_{j}^{K, i}\left(\xi_{k}^{K, i}\right)=\delta_{j k}$.

Definition 5.11 (Flux reconstruction). Let $\widehat{\boldsymbol{f}}_{1} \in \mathbb{V}_{q+1, q}$ satisfy

$$
\int_{\mathscr{T}}\left(\partial_{x_{1}} \widehat{\boldsymbol{f}}_{1}\right) \Phi=-\int_{\mathscr{T}} \boldsymbol{f}_{1}\left(\boldsymbol{v}_{h}\right) \partial_{x_{1}} \Phi+\int_{\mathscr{E} V} \mathscr{P}_{q}\left[\boldsymbol{F}_{1}\left(\boldsymbol{v}_{h}^{-}, \boldsymbol{v}_{h}^{+}\right)\right] \llbracket \Phi \rrbracket^{h} \quad \forall \Phi \in \mathbb{V}_{q, q},
$$

where $\mathscr{P}_{q}$ denotes $\mathrm{L}_{2}$-orthogonal projection in the space of piece-wise polynomials of degree $\leq q$ on $\mathscr{E}^{v}$, and

$$
\widehat{\boldsymbol{f}}_{1}\left(x_{i}, \xi_{k}^{K, 2}\right)^{+}:=\lim _{s \searrow 0} \widehat{\boldsymbol{f}}_{1}\left(x_{i}+s, \xi_{k}^{K, 2}\right)=\mathscr{P}_{q}\left[\boldsymbol{F}_{1}\left(\boldsymbol{v}_{h}^{-}, \boldsymbol{v}_{h}^{+}\right)\right]\left(x_{i}, \xi_{k}^{K, 2}\right)
$$

for $k=0, \ldots, q$ and all $K \in \mathscr{T}$. The definition of $\widehat{\boldsymbol{f}}_{2} \in \mathbb{V}_{q, q+1}$ is analogous.

Remark 5.12 (Regularity of flux reconstruction). Note that in order to split the residual in two space dimensions in a way analogous to what we did in (5.16) we require that for $\alpha=1,2$ the components of the flux reconstruction $\widehat{f}_{\alpha}$ are Lipschitz continuous in $x_{\alpha}$-direction. This is exactly what is needed such that $\partial_{x_{\alpha}} \widehat{\boldsymbol{f}}_{\alpha}$ makes sense in $\mathrm{L}_{\infty}$.

Lemma 5.13 (Properties of flux reconstruction). The flux reconstructions $\widehat{\boldsymbol{f}}_{1}, \widehat{\boldsymbol{f}}_{2}$ are well defined; and $\widehat{\boldsymbol{f}}_{1}$ is Lipschitz continuous in $x_{1}$-direction and $\widehat{\boldsymbol{f}}_{2}$ is Lipschitz continuous in $x_{2}$-direction.

Proof. We will give the proof for $\widehat{\boldsymbol{f}}_{1}$. For every $K \in \mathscr{T}$ the restriction $\left.\widehat{\boldsymbol{f}}_{1}\right|_{K}$ is determined by (5.19) up to a linear combination (in each component) of

$$
1 \otimes l_{0}^{K, 2}, \ldots, 1 \otimes l_{q}^{K, 2},
$$

where 1 denotes the polynomial having the value 1 everywhere. Prescribing (5.20) obviously fixes these degrees of freedom. Therefore, $\widehat{f}_{1}$ exists, is uniquely determined, and locally computable.

For showing that $\widehat{\boldsymbol{f}}_{1}$ is Lipschitz in the $x_{1}$-direction it suffices to prove that $\hat{\boldsymbol{f}}_{1}$ is continuous along the 'vertical' faces. Let $K=\left[x_{i}, x_{i+1}\right] \times\left[y_{j}, y_{j+1}\right] \in \mathscr{T}$ then we define

$$
\chi_{K}^{k}:=1_{\left[x_{i}, x_{i+1}\right]} \otimes\left(l_{k}^{K, 2} \cdot 1_{\left[y_{j}, y_{j+1}\right]}\right)
$$

where for any interval $I$ we denote the characteristic function of that interval by $1_{I}$. For any $k \in\{0, \ldots, q\}$ we have on the one hand

$$
\int_{\mathscr{T}} \partial_{x_{1}} \widehat{\boldsymbol{f}}_{1} \chi_{K}^{k}=\omega_{k} h_{j}^{y} \int_{x_{i}}^{x_{i+1}} \partial_{x_{1}} \widehat{\boldsymbol{f}}_{1}\left(\cdot, \xi_{k}^{K, 2}\right)=\omega_{k} h_{j}^{y}\left(\widehat{\boldsymbol{f}}_{1}\left(x_{i+1}, \xi_{k}^{K, 2}\right)^{-}-\widehat{\boldsymbol{f}}_{1}\left(x_{i}, \xi_{k}^{K, 2}\right)^{+}\right)
$$


where $h_{j}^{y}=y_{j+1}-y_{j}$ and $\omega_{k}$ is the Gauss quadrature weight associated to $\xi_{k}$. On the other hand we find, using (5.19),

$$
\int_{\mathscr{T}} \partial_{x_{1}} \widehat{\boldsymbol{f}}_{1} \chi_{K}^{k}=\omega_{k} h_{j}^{y}\left(\mathscr{P}_{q}\left[\boldsymbol{F}_{1}\left(\boldsymbol{v}_{h}^{-}, \boldsymbol{v}_{h}^{+}\right)\right]\left(x_{i+1}, \xi_{k}^{K, 2}\right)-\mathscr{P}_{q}\left[\boldsymbol{F}_{1}\left(\boldsymbol{v}_{h}^{-}, \boldsymbol{v}_{h}^{+}\right)\right]\left(x_{i}, \xi_{k}^{K, 2}\right)\right) .
$$

Combining (5.21), (5.22) and (5.20) we obtain

$$
\widehat{\boldsymbol{f}}_{1}\left(x_{i+1}, \xi_{k}^{K, 2}\right)^{-}=\mathscr{P}_{q}\left[\boldsymbol{F}_{1}\left(\boldsymbol{v}_{h}^{-}, \boldsymbol{v}_{h}^{+}\right)\right]\left(x_{i+1}, \xi_{k}^{K, 2}\right)=\widehat{\boldsymbol{f}}_{1}\left(x_{i+1}, \xi_{k}^{K, 2}\right)^{+} \quad \text { for } k=0, \ldots, q .
$$

As $\left.\widehat{\boldsymbol{f}}_{1}\left(x_{i+1}, \cdot\right)^{ \pm}\right|_{\left[y_{j}, y_{j+1}\right]}$ is a polynomial of degree $q$ and $k$ is arbitrary in equation (5.23) we find

$$
\left.\widehat{\boldsymbol{f}}_{1}\left(x_{i+1}, \cdot\right)^{+}\right|_{\left[y_{j}, y_{j+1}\right]}=\left.\widehat{\boldsymbol{f}}_{1}\left(x_{i+1}, \cdot\right)^{-}\right|_{\left[y_{j}, y_{j+1}\right]} .
$$

As $i, j$ were arbitrary this implies Lipschitz continuity of $\widehat{f}_{1}$ in $x_{1}$-direction.

From equations (5.18) and (5.19) we obtain the following pointwise equation almost everywhere:

$$
\partial_{t} \boldsymbol{v}_{h}+\partial_{x_{1}} \widehat{\boldsymbol{f}}_{1}+\partial_{x_{2}} \widehat{\boldsymbol{f}}_{2}=0 .
$$

Remark 5.14 (Main idea of a 2 dimensional reconstruction). Recalling the arguments presented in previous Sections our main priority is to make $\widehat{\boldsymbol{v}}$ Lipschitz continuous. The particular reconstruction we describe is based on the following principles inspired by $\S 5.1$. We wish $\left.\widehat{\boldsymbol{v}}\right|_{K}-\left.\boldsymbol{v}_{h}\right|_{K}$ to be orthogonal to polynomials on $K$ of degree $q-1$ which is ensured by imposing them to coincide on the tensor product Gauss points. We wish $\widehat{\boldsymbol{f}}_{1}$ and $\boldsymbol{f}_{1}(\widehat{\boldsymbol{v}})$ to be similar on vertical faces which is ensured by fixing the values of $\widehat{\boldsymbol{v}}$ on points of the form $\left(x_{i}, \xi_{l}^{K, 2}\right)$ when $K=\left[x_{i}, x_{i+1}\right] \times\left[y_{j}, y_{j+1}\right]$. Imposing the conditions described above on a reconstruction in $\mathbb{V}_{q+1, q+1}$ is impossible because it does not have enough degrees of freedom. Thus, we define a reconstruction $\widehat{\boldsymbol{v}} \in \mathbb{V}_{q+2, q+2}$. For such a function imposing the degrees of freedom described above leaves four degrees of freedom per cell undefined. Thus, we may prescribe values in corners. To this end let us fix an averaging operator $\overline{\boldsymbol{w}}: U^{4} \rightarrow U$.

Definition 5.15 (Solution reconstruction). We define (at each time) the reconstruction $\widehat{\boldsymbol{v}} \in \mathbb{V}_{q+2, q+2}$ of $\boldsymbol{v}_{h} \in \mathbb{V}_{q, q}$ by prescribing for every $K=\left[x_{i}, x_{i+1}\right] \times\left[y_{j}, y_{j+1}\right] \in \mathscr{T}$

$$
\begin{aligned}
&\left.\widehat{\boldsymbol{v}}\right|_{K}\left(\xi_{k}^{K, 1}, \xi_{l}^{K, 2}\right)=\boldsymbol{v}_{h}\left(\xi_{k}^{K, 1}, \xi_{l}^{K, 2}\right) \quad \text { for } k, l=0, \ldots, q \\
&\left.\widehat{\boldsymbol{v}}\right|_{K}\left(x_{i}, \xi_{l}^{K, 2}\right)=\boldsymbol{w}_{1}\left(\boldsymbol{v}_{h}\left(x_{i}, \xi_{l}^{K, 2}\right)^{-}, \boldsymbol{v}_{h}\left(x_{i}, \xi_{l}^{K, 2}\right)^{+}\right) \quad \text { for } l=0, \ldots, q \\
&\left.\widehat{\boldsymbol{v}}\right|_{K}\left(x_{i+1}, \xi_{l}^{K, 2}\right)=\boldsymbol{w}_{1}\left(\boldsymbol{v}_{h}\left(x_{i+1}, \xi_{l}^{K, 2}\right)^{-}, \boldsymbol{v}_{h}\left(x_{i+1}, \xi_{l}^{K, 2}\right)^{+}\right) \quad \text { for } l=0, \ldots, q \\
&\left.\widehat{\boldsymbol{v}}\right|_{K}\left(\xi_{k}^{K, 1}, y_{j}\right)=\boldsymbol{w}_{2}\left(\boldsymbol{v}_{h}\left(\xi_{k}^{K, 1}, y_{j}\right)^{-}, \boldsymbol{v}_{h}\left(\xi_{k}^{K, 1}, y_{j}\right)^{+}\right) \quad \text { for } k=0, \ldots, q \\
&\left.\widehat{\boldsymbol{v}}\right|_{K}\left(\xi_{k}^{K, 1}, y_{j+1}\right)=\boldsymbol{w}_{2}\left(\boldsymbol{v}_{h}\left(\xi_{k}^{K, 1}, y_{j+1}\right)^{-}, \boldsymbol{v}_{h}\left(\xi_{k}^{K, 1}, y_{j+1}\right)^{+}\right) \quad \text { for } k=0, \ldots, q \\
&\left.\widehat{\boldsymbol{v}}\right|_{K}\left(x_{i}, y_{j}\right)=\overline{\boldsymbol{w}}\left(\lim _{s \searrow 0} \boldsymbol{v}_{h}\left(x_{i}+s, y_{j}+s\right), \lim _{s \searrow 0} \boldsymbol{v}_{h}\left(x_{i}-s, y_{j}+s\right),\right. \\
&\left.\lim _{s \searrow 0} \boldsymbol{v}_{h}\left(x_{i}+s, y_{j}-s\right), \lim _{s \searrow 0} \boldsymbol{v}_{h}\left(x_{i}-s, y_{j}-s\right)\right)
\end{aligned}
$$

and analogous prescriptions for the remaining three corners of $K$.

Lemma 5.16 (Properties of $\widehat{\boldsymbol{v}}$ ). The reconstruction $\widehat{\boldsymbol{v}}$, is well-defined, locally computable and Lipschitz continuous. Moreover, for $q \geq 1$ the following local conservation property is satisfied:

$$
\int_{K} \widehat{\boldsymbol{v}}-\boldsymbol{v}_{h}=0 \quad \forall K \in \mathscr{T} .
$$

Proof. We will only prove the Lipschitz continuity and the conservation property. As $\widehat{\boldsymbol{v}}$ is piecewise polynomial it is sufficient to prove continuity to show Lipschitz continuity. Let $K=\left[x_{i}, x_{i+1}\right] \times\left[y_{j}, y_{j+1}\right]$ and $K^{\prime}=\left[x_{i-1}, x_{i}\right] \times\left[y_{j}, y_{j+1}\right]$ then $\left.\widehat{\boldsymbol{v}}\right|_{K}$ and $\left.\widehat{\boldsymbol{v}}\right|_{K^{\prime}}$ coincide on $\left(x_{i}, y_{j}\right),\left(x_{i}, \xi_{k}^{K, 2}\right)_{k=0, \ldots, q}$ and $\left(x_{i}, y_{j+1}\right)$. Therefore, $\left.\widehat{\boldsymbol{v}}\right|_{K}$ and $\left.\widehat{\boldsymbol{v}}\right|_{K^{\prime}}$ coincide on $\left\{x_{i}\right\} \times\left[y_{j}, y_{j+1}\right]$. Analogous arguments hold for the other edges, such that $\widehat{\boldsymbol{v}}$ is indeed (Lipschitz) continuous.

As the nodal points on each element have tensor structure we can use the exactness properties of onedimensional Gauss quadrature. The conservation property (5.27) follows from the fact that one-dimensional Gauss quadrature with $q+1$ Gauss points is exact for polynomials of degree up to $2 q+1$ which is larger or equal $q+2$ provided $q \geq 1$. 
Remark 5.17 (Reconstructions for hyperbolic/parabolic problems in 2 dimensions). In order to obtain reconstructions and splittings of residuals into hyperbolic and parabolic parts for numerical discretisations of (5.1) the reconstructions $\widehat{\boldsymbol{v}}, \widehat{\boldsymbol{f}}_{\alpha}$ described in this section can be used in the same way the reconstructions from $\S 5.1$ were used in $\S 5.2$. In particular, $\widehat{\boldsymbol{v}}$ described above is already regular enough to serve as a reconstruction in case of a numerical scheme for (5.1). The flux reconstructions $\left(\widehat{f}_{\alpha}\right)_{\alpha=1,2}$ can be used to obtain a splitting analogous to (5.16) by making use of (5.25).

\section{NUMERICAL EXPERIMENTS}

In this section we study the numerical behaviour of the error indicators $\mathscr{E}_{M}$ and $\mathscr{E}_{D}$ presented in the previous Sections and compare this with the "error", which we quantify as the difference between the numerical approximation of the adaptive model and the numerical approximation of the full model, on some test problems.

The model adaptive algorithm we employ is encapsulated by the following pseudocode:

\subsection{Model Adaptation.}

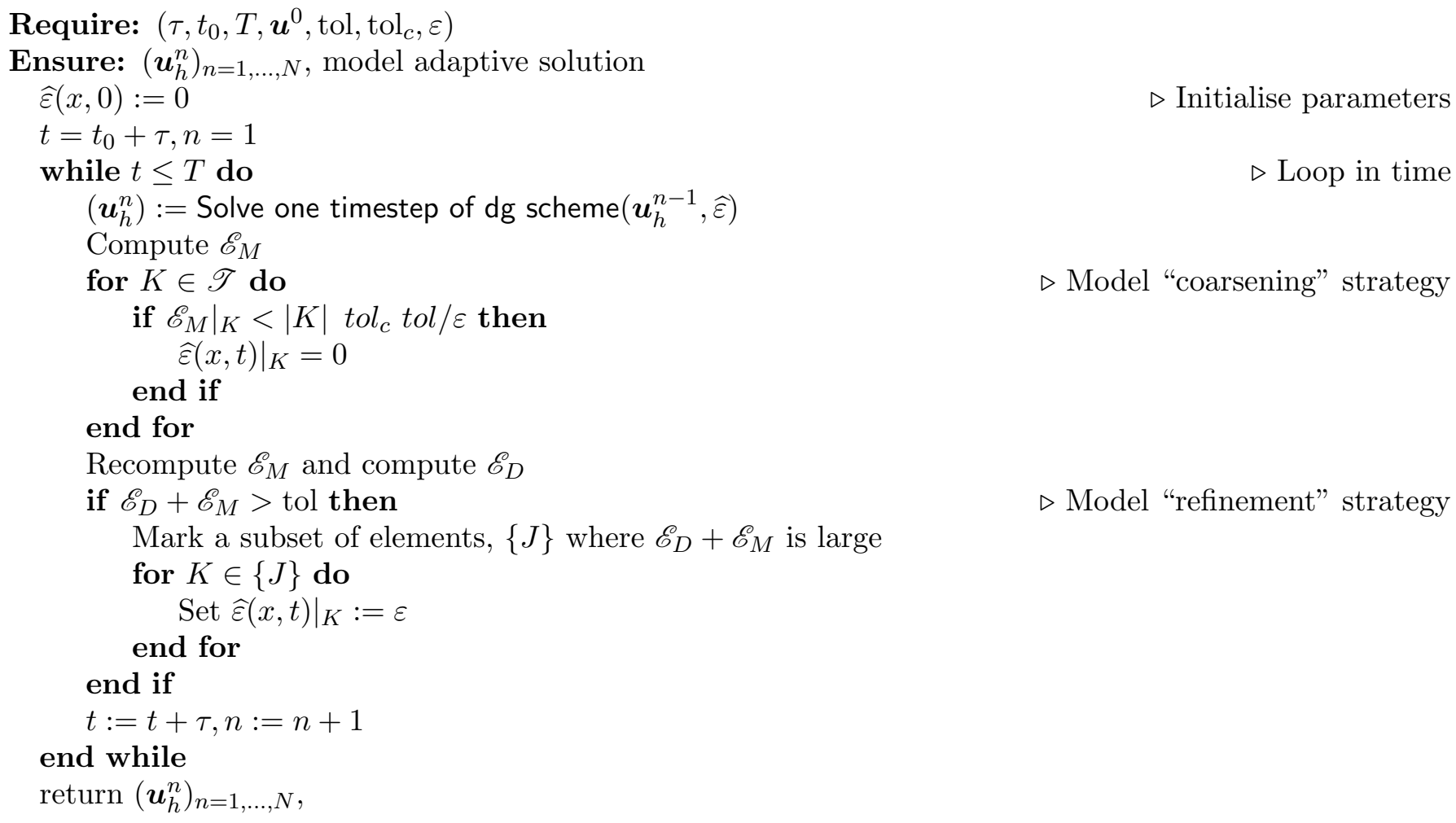

Remark 6.1 (Coupling to other adaptive procedures). The a posteriori bound given in Theorem 4.2 has a structure which allows for both model and mesh adaptivity. This means that Algorithm 6.1 could be coupled with other mechanisms employing $h-p$ spatial adaptivity in addition to local timestep control. As can be seen from the pseudocode we use the complex model even in the case the discretisation error $\mathscr{E}_{D}$ is large and the modelling error $\mathscr{E}_{M}$ is small. In the first few tests we focus on the effect of model adaptation only and will demonstrate one possibility of coupling model and mesh adaptivity in the final test.

6.2. Test 1 : The scalar case - the 1d viscous and inviscid Burgers' equation. We conduct an illustrative experiment using Burgers' equation. In this case the "complex" model which we want to approximate is given by

$$
\partial_{t} u_{\varepsilon}+\partial_{x}\left(\frac{u_{\varepsilon}^{2}}{2}\right)=\varepsilon \partial_{x x} u_{\varepsilon}
$$

for fixed $\varepsilon=0.005$ with homogeneous Dirichlet boundary data and the "simple" model we will use in the majority of the domain is given by

$$
\partial_{t} u+\partial_{x}\left(\frac{u^{2}}{2}\right)=0 .
$$


We discretise the problem (6.2) using a piecewise linear dG scheme (5.5) together with Richtmyer type fluxes given by

$$
F\left(v_{h}^{-}, v_{h}^{+}\right)=f\left(\frac{1}{2}\left(v_{h}^{-}+v_{h}^{+}\right)-\frac{\tau}{h}\left(f\left(v_{h}^{+}\right)-f\left(v_{h}^{-}\right)\right)\right) .
$$

Note that these fluxes satisfy the assumptions (5.6)-(5.7). The dG formulation is then given by (5.5). In the region where the "complex" model (6.1) is implemented for the discretisation of the diffusion term we use an interior penalty (IP) discretisation with piecewise constant $\widehat{\varepsilon}$, that is

$$
\widehat{\varepsilon}=\left\{\begin{array}{l}
0.005 \text { over cells where the a posteriori model error bound is large } \\
0 \text { otherwise. }
\end{array}\right.
$$

This means the discretisation becomes

$$
\begin{gathered}
\int_{\mathscr{T}} \partial_{t} u_{h} \cdot \phi-f\left(u_{h}\right) \cdot \partial_{x} \phi+\int_{\mathscr{E}} F\left(u_{h}^{-}, u_{h}^{+}\right) \llbracket \phi \rrbracket+\mathscr{A}_{h}\left(u_{h}, \phi ; \widehat{\varepsilon}\right)=0 \quad \text { for all } \phi \in \mathbb{V}_{q}, \\
u_{h}(0)=\mathscr{P}_{q}\left[u_{0}\right]
\end{gathered}
$$

where

$$
\mathscr{A}_{h}\left(w_{h}, \phi ; \widehat{\varepsilon}\right)=\int_{\mathscr{T}} \widehat{\varepsilon} \partial_{x} w_{h} \cdot \partial_{x} \phi-\int_{\mathscr{E}} \llbracket w_{h} \rrbracket \cdot\left\{\widehat{\varepsilon} \partial_{x} \phi\right\}+\llbracket \phi \rrbracket \cdot\left\{\widehat{\varepsilon} \partial_{x} w_{h}\right\}-\frac{\sigma(\epsilon)}{h} \llbracket w_{h} \rrbracket \cdot \llbracket \phi \rrbracket
$$

and $\sigma(\epsilon)=10 \epsilon$ is the penalty parameter. Initial conditions are chosen as

$$
u(x, 0):=\sin (x)
$$

over the interval $[-\pi, \pi]$. We use a first order IMEX temporal discretisation where the diffusion is taken implicitly and the other terms explicitly. We take $\tau=10^{-4}$ and $h=\pi / 500$ uniformly over the space-time domain. For this test the parameters for Algorithm 6.1 are tol $=10^{-2}$ and $\operatorname{tol}_{c}=10^{-3}$. Note that these are user-specified parameters. The results are summarised in Figures 1 and 2. In Figure 1 we show snapshots of the solution over time together with the value of the model adaptivity parameter. In Figure 2 we display the error induced by solving the "complex" model only over part of the domain.

6.3. Test 2 : The scalar case - the $2 \mathrm{~d}$ viscous and inviscid Burgers' equation. In this test we examine how the adaptive procedure extends into the multi-dimensional setting again using Burgers' equation as an illustrative example. In this case the "complex" model which we want to approximate is given by

$$
\partial_{t} u_{\varepsilon}+\operatorname{div}\left(\frac{\mathbb{1} u_{\varepsilon}^{2}}{2}\right)=\varepsilon \Delta u_{\varepsilon}
$$

where $\mathbb{1}=(1,1)^{\boldsymbol{\top}}$. The simple model we will use in the majority of the domain is given by

$$
\partial_{t} u+\operatorname{div}\left(\frac{\mathbb{1} u^{2}}{2}\right)=0 .
$$

These are coupled with homogenous Dirichlet boundary conditions. As in Test 1 we make use of a 1st order IMEX, piecewise linear dG scheme together with Richtmyer fluxes and an IP method for the viscosity. We pick an initial condition

$$
u(\boldsymbol{x}, 0)=\exp \left(-10|\boldsymbol{x}|^{2}\right)
$$

and use the parameters $\varepsilon=0.01, h=\sqrt{2} / 50, \tau=\sqrt{2} / 400$, tol $=10^{-2}$ and tol ${ }_{c}=10^{-3}$ in Algorithm 6.1. The results are summarised in Figures 3 and 4. In Figure 3 snapshots of the solution over time together with the value of the model adaptivity parameter are shown. Figure 4 displays the error induced by solving the "complex" model only over part of the domain. 
Figure 1. A numerical experiment testing model adaptivity on Burgers' equation. The simulation is described in $\S 6.2$. Here we display the solution at various times (top) together with a representation of the model adaptation parameter $\widehat{\varepsilon}$ (bottom). Blue is the region $\widehat{\varepsilon}=0$, where the simplified (inviscid Burgers') problem is being computed and red is where $\widehat{\varepsilon}=\varepsilon \neq 0$, where the full (viscous Burgers') problem is being computed. We see that initially only the simplified model is computed but as time progresses the full model is solved in a region around where the steep layer forms. As this forms the domain where the complex model is solved collapses and eventually is very localised around the layer.

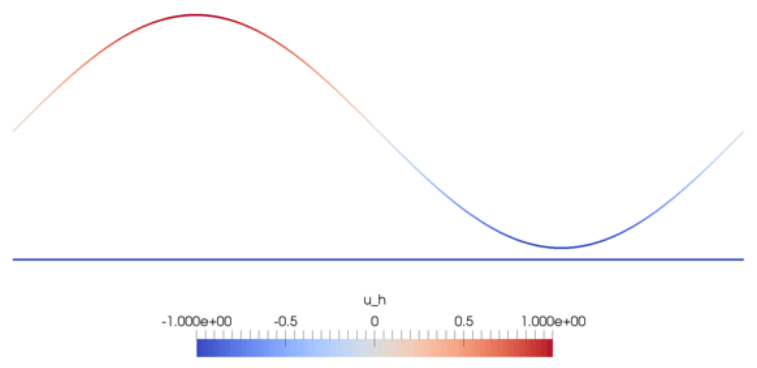

(a) $t=0$

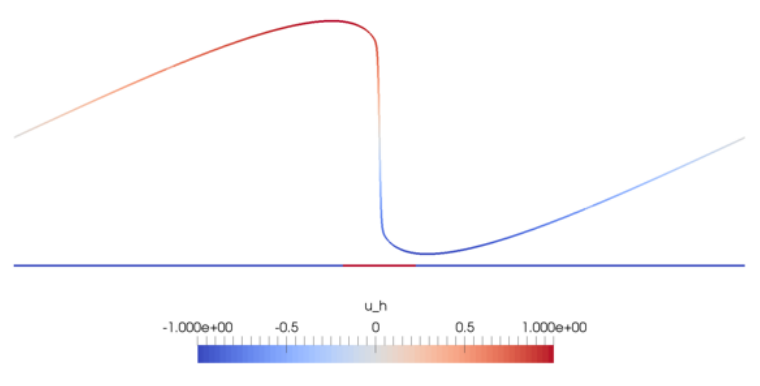

(c) $t=1.1625$

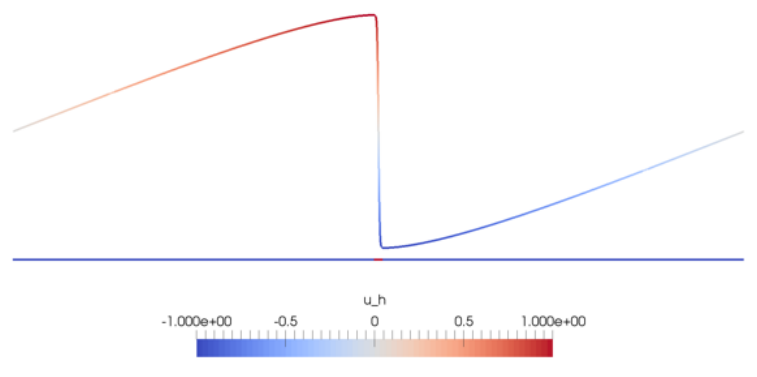

(e) $t=1.55$

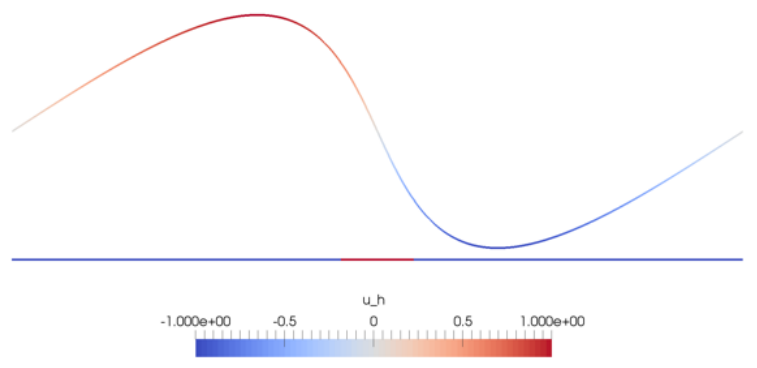

(b) $t=0.5375$

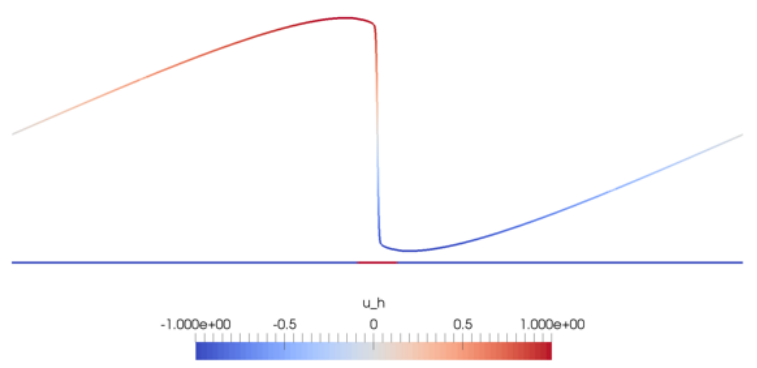

(d) $t=1.3$

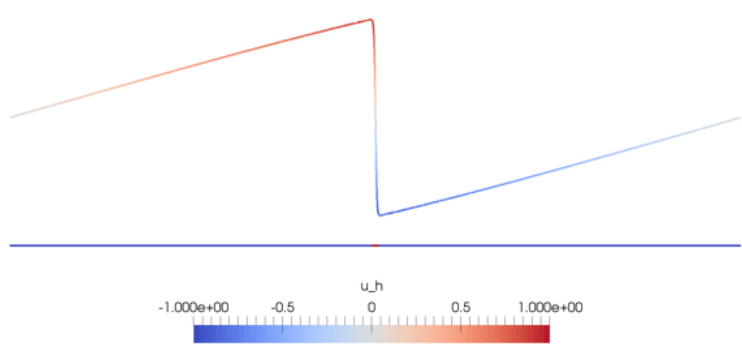

(f) $t=2.5$ 
Figure 2. A numerical experiment testing model adaptivity on Burgers' equation. The simulation is described in $\S 6.2$. Here we display the error $\left|u_{h}-u_{\varepsilon, h}\right|$, that is, the difference between the approximation of the full expensive model and that of the adaptive approximation at the same times as in Figure 1 together with a representation of $\widehat{\varepsilon}$ (bottom). An interesting phenomenon is the propagation of dispersive waves emanating from the interface between the region where $\widehat{\varepsilon}=0$ and that of $\widehat{\varepsilon}=\varepsilon \neq 0$.

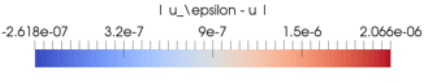

(a) $t=0$

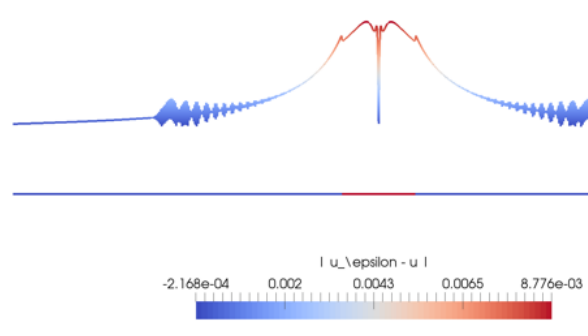

(c) $t=1.1625$
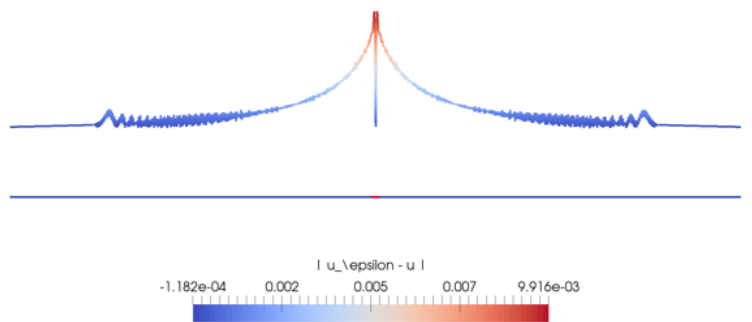

(e) $t=1.55$
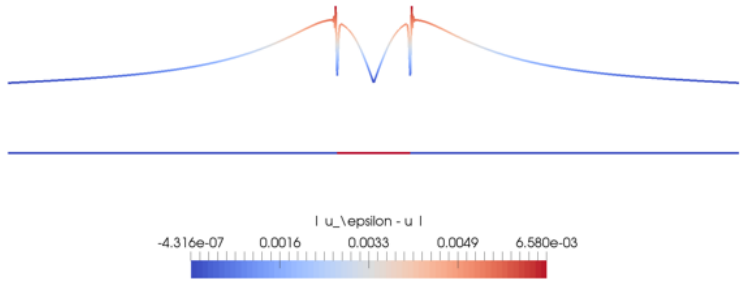

(b) $t=0.5375$
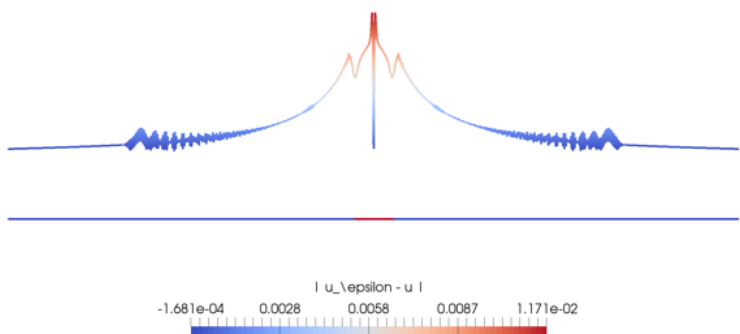

(d) $t=1.3$
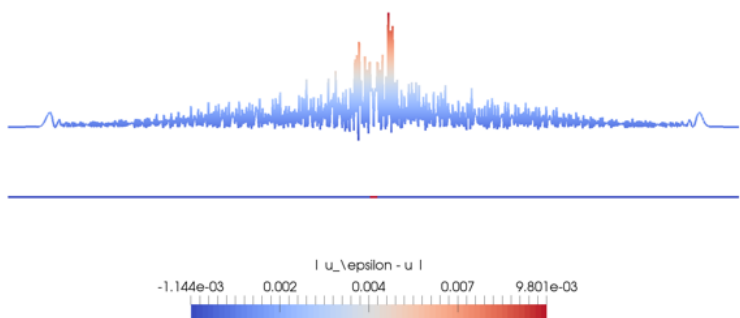

(f) $t=2.5$ 
Figure 3. A numerical experiment testing model adaptivity on Burgers' equation. The simulation is described in $\S 6.3$. Here we display the solution at various times (top) together with a representation of $\widehat{\varepsilon}$ (bottom). Blue is the region $\widehat{\varepsilon}=0$, where the simplified (inviscid Burgers') problem is being computed and red is where $\widehat{\varepsilon}=\varepsilon \neq 0$, where the full (viscous Burgers') problem is being computed.

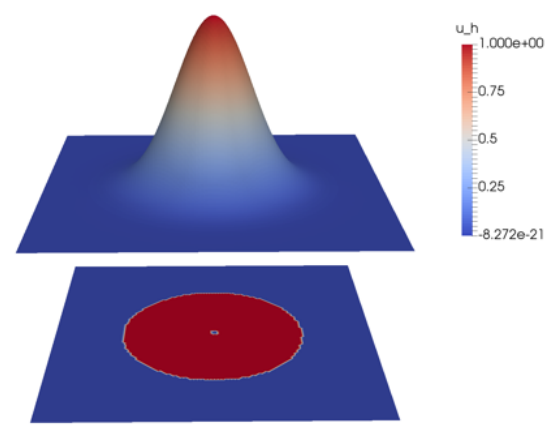

(a) $t=0.0025$

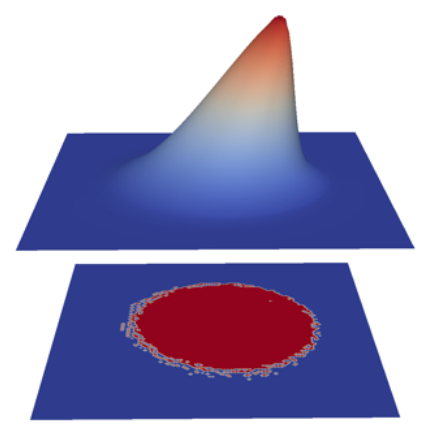

(c) $t=0.5$

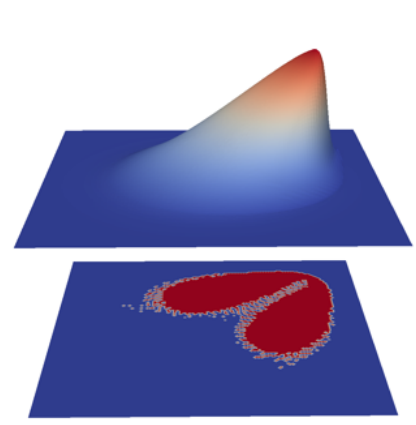

(e) $t=1.25$

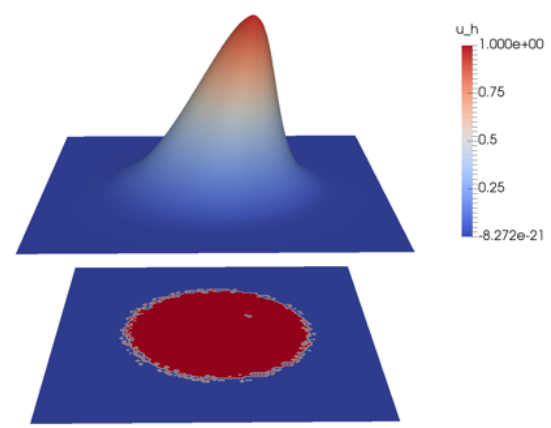

(b) $t=0.25$

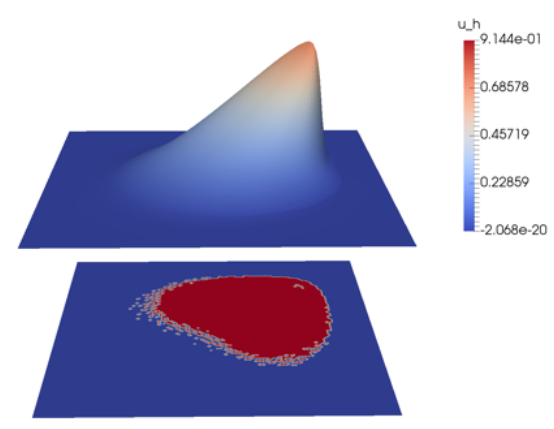

(d) $t=1$.

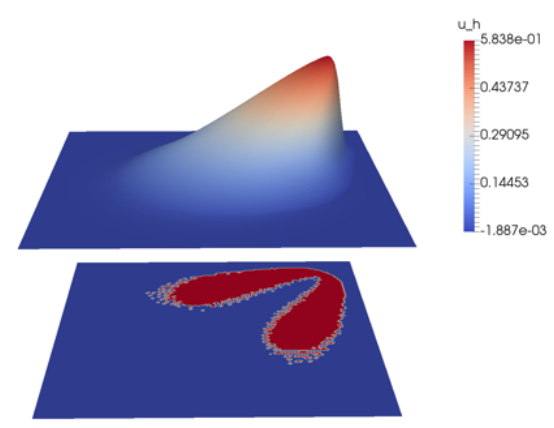

(f) $t=1.5$ 
Figure 4. A numerical experiment testing model adaptivity on Burgers' equation. The simulation is described in $\S 6.3$. Here we display the error $\left|u_{h}-u_{\varepsilon, h}\right|$, that is, the difference between the approximation of the full expensive model and that of the adaptive approximation at the same times as in Figure 3.

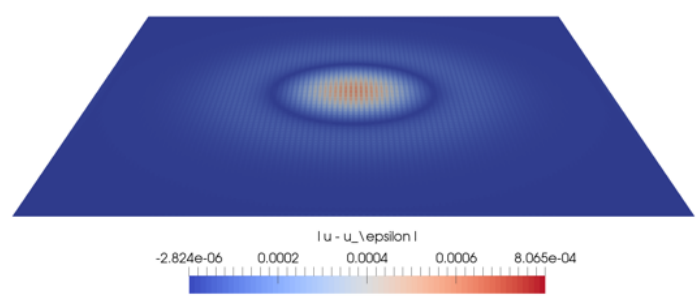

(a) $t=0.0025$

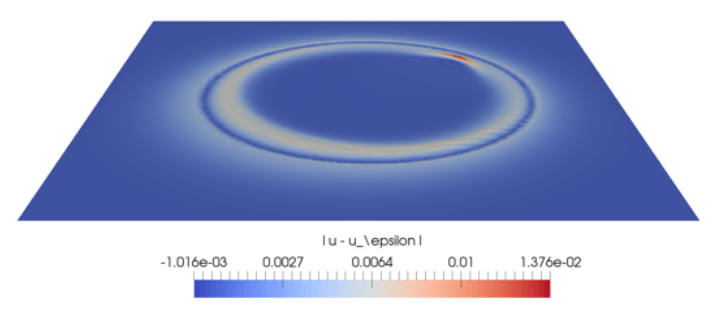

(c) $t=0.5$

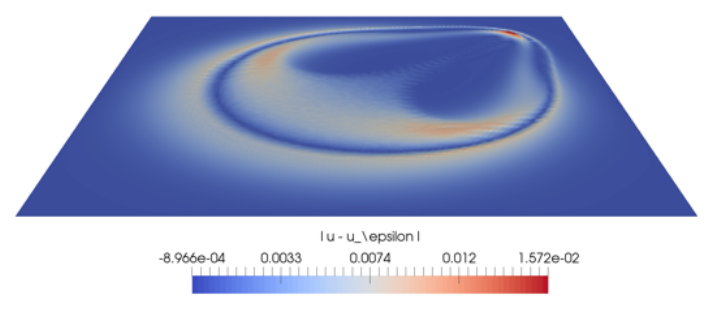

(e) $t=1.25$

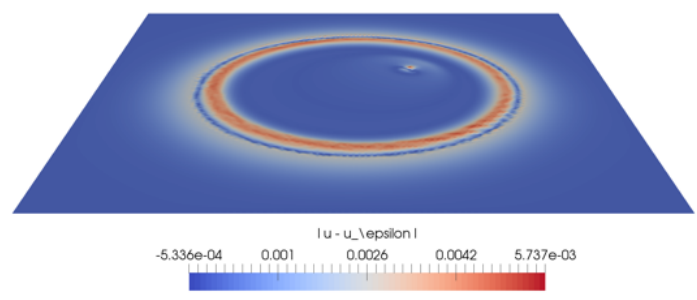

(b) $t=0.25$

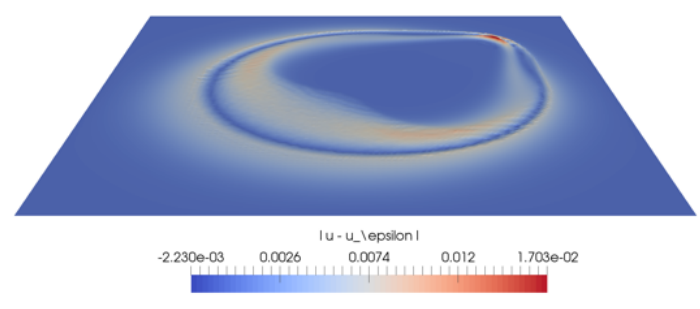

(d) $t=1$.

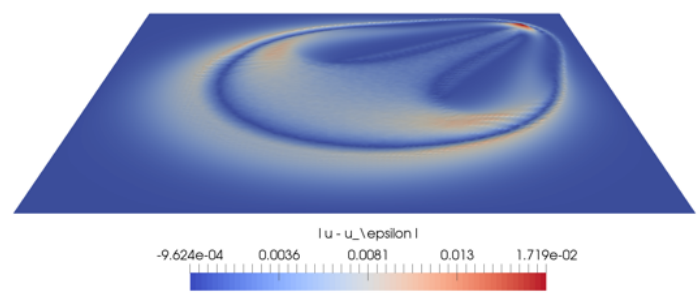

(f) $t=1.5$ 
6.4. Test 3 : The Isothermal Navier-Stokes system. In this test we examine the use of model adaptivity on the Isothermal Navier-Stokes system in a situation where Kármán vortices are produced by a flow over a cylinder. This can be achieved by seeking $\left(\rho_{\mu}, \rho \mathbf{v}_{\mu}\right)$ such that

$$
\begin{aligned}
\partial_{t} \rho_{\mu}+\operatorname{div}\left(\rho_{\mu} \mathbf{v}_{\mu}\right) & =0 \\
\partial_{t}\left(\rho_{\mu} \mathbf{v}_{\mu}\right)+\operatorname{div}\left(\rho_{\mu} \mathbf{v}_{\mu} \otimes \mathbf{v}_{\mu}\right)+\nabla p & =\operatorname{div}\left(\mu \nabla \mathbf{v}_{\mu}\right),
\end{aligned}
$$

with a Reynolds number of 100 (i.e., $\mu=\frac{1}{100}$ ). In this case our "complex" model is given by (6.11) and the approximation is given by

$$
\begin{aligned}
\partial_{t} \rho+\operatorname{div}(\rho \mathbf{v}) & =0 \\
\partial_{t}(\rho \mathbf{v})+\operatorname{div}(\rho \mathbf{v} \otimes \mathbf{v})+\nabla p & =0 .
\end{aligned}
$$

Here we use the relation $p(\rho)=0.2 \rho$, through Boyle's law. We write $\boldsymbol{w}=\left(\rho_{\mu}, \rho_{\mu} \mathbf{v}_{\mu}\right)$ for the unknowns and then (6.11) becomes

$$
\partial_{t} \boldsymbol{w}+\operatorname{div}(\boldsymbol{f}(\boldsymbol{w}))=\boldsymbol{D}(\boldsymbol{w})
$$

with

$$
\boldsymbol{D}(\boldsymbol{w})=\left[\begin{array}{c}
0 \\
\operatorname{div}\left(\mu \nabla \mathbf{v}_{\mu}\right)
\end{array}\right]
$$

representing the diffusion term. We choose a piecewise linear dG scheme similar to that in previous tests of the form

$$
\begin{aligned}
\int_{\mathscr{T}} \partial_{t} \boldsymbol{w}_{h} \cdot \boldsymbol{\phi}-\boldsymbol{f}\left(\boldsymbol{w}_{h}\right): \nabla \boldsymbol{\phi}+\int_{\mathscr{E}} \boldsymbol{F}\left(\boldsymbol{w}_{h}^{-}, \boldsymbol{w}_{h}^{+}\right) \llbracket \phi \rrbracket & =\mathscr{D}\left(\boldsymbol{w}_{h}, \boldsymbol{\phi} ; \widehat{\mu}\right) \quad \text { for all } \boldsymbol{\phi} \in \mathbb{V}_{q}, \\
u_{h}(0) & =\mathscr{P}_{q}\left[u_{0}\right]
\end{aligned}
$$

where, in $2 d$,

$$
\boldsymbol{f}(\boldsymbol{w})=\left[\begin{array}{cc}
\rho v_{1} & \rho v_{2} \\
\rho v_{1}^{2}+p & \rho v_{1} v_{2} \\
\rho v_{2} v_{1} & \rho v_{2}^{2}+p
\end{array}\right],
$$

the div operator is understood to act row-wise,

$$
\mathscr{D}\left(\boldsymbol{w}_{h}, \boldsymbol{\phi} ; \widehat{\mu}\right)=\left(\begin{array}{c}
0 \\
-\mathscr{A}_{h}\left(v_{1}, \phi_{2} ; \widehat{\mu}\right) \\
-\mathscr{A}_{h}\left(v_{2}, \phi_{3} ; \widehat{\mu}\right)
\end{array}\right),
$$

and

$$
\mathscr{A}_{h}(v, \phi ; \widehat{\mu})=\int_{\mathscr{T}} \widehat{\mu} \nabla v \cdot \nabla \phi-\int_{\mathscr{E}} \llbracket v \rrbracket \cdot\{\widehat{\mu} \nabla \phi\}+\llbracket \phi \rrbracket \cdot\{\widehat{\mu} \nabla v\}-\frac{\sigma(\mu)}{h} \llbracket v \rrbracket \cdot \llbracket \phi \rrbracket
$$

with $\sigma(\mu)=10 \mu$ as the penalty parameter.

As in the previous tests we make use of a first order IMEX scheme for the timestepping and Richtmyer fluxes for $\boldsymbol{F}$. We take the domain as a rectangular region $[-1,7.5] \times[-0.7,0.7]$ with a circular hole centered at the origin of radius 0.05 . In this case $\max h_{K} \approx 0.14$ occuring near the upper and lower boundaries and $\min h_{K} \approx 0.00008$ occuring near the hole. The initial data we use is

$$
\rho(\boldsymbol{x}, 0)=1 \quad \boldsymbol{v}(\boldsymbol{x}, 0)=(1,0)^{\boldsymbol{\top}} .
$$

We impose slip boundary conditions on the top and bottom of the rectangular region, an inflow and outflow on the left and right hand side respectively, compatible with the initial conditions and a no slip condition on the cylinder itself.

The results are given in Figure 5 which shows the vorticity, $\omega=\partial_{x} v_{2}-\partial_{y} v_{1}$, of the simulation with model adaptivity and the location of where diffusion was switched on. Figure 6 gives an indication of the qualitative difference between the adaptive approximation and the simulation of the full INS system for $T=6.02$. 
Figure 5. A numerical experiment testing model adaptivity on the Isothermal NavierStokes system. The simulation is described in $\S 6.4$. Here we display the vorticity, $\omega=$ $\partial_{x} v_{2}-\partial_{y} v_{1}$, of the solution at various times (top) together with a representation of both $\mu$ (bottom). Blue is the region $\mu=0$, where the simplified (Euler system) problem is being computed and red is where $\mu \neq 0$, where the full (Isothermal Navier-Stokes system) problem is being computed.

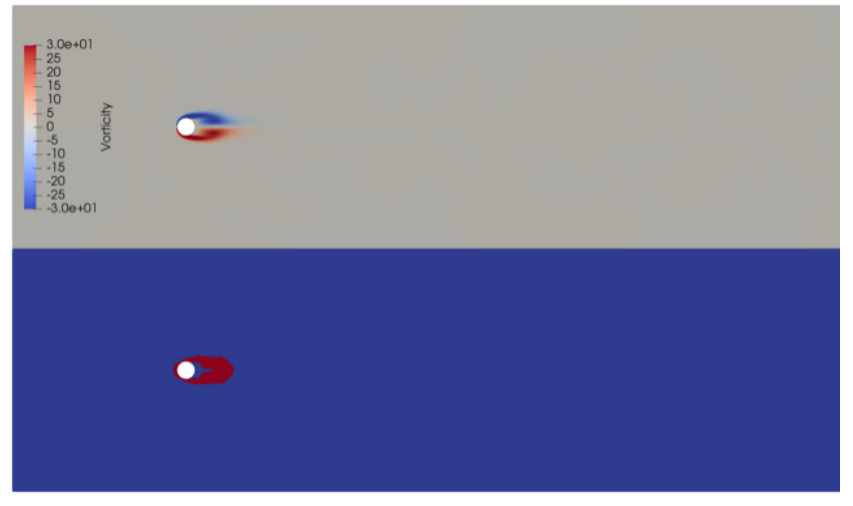

(a) $t=0.04$

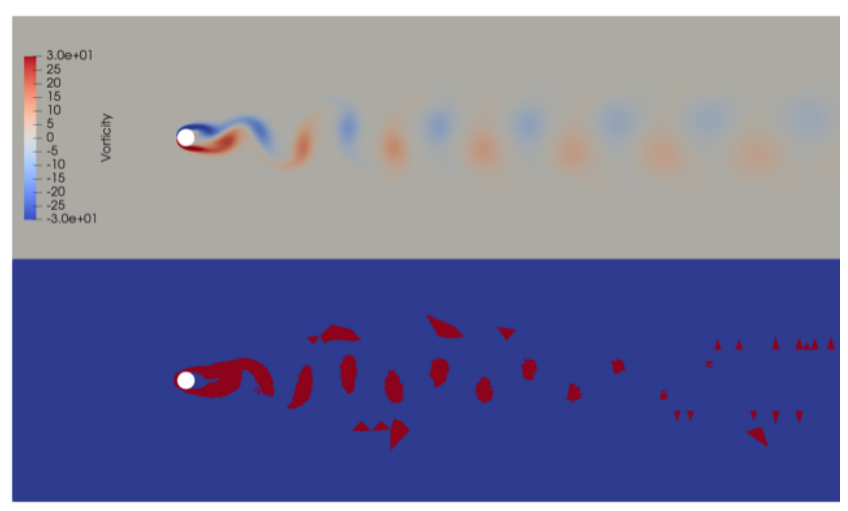

(c) $t=0.94$

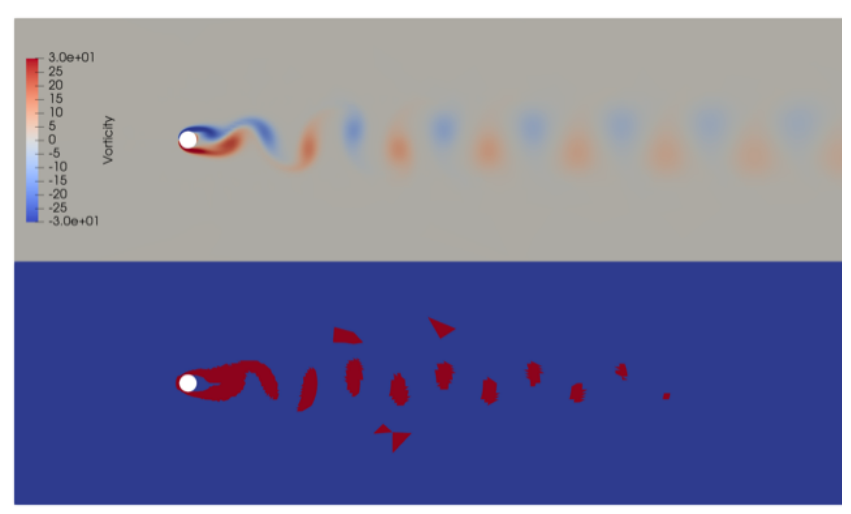

(e) $t=2.46$

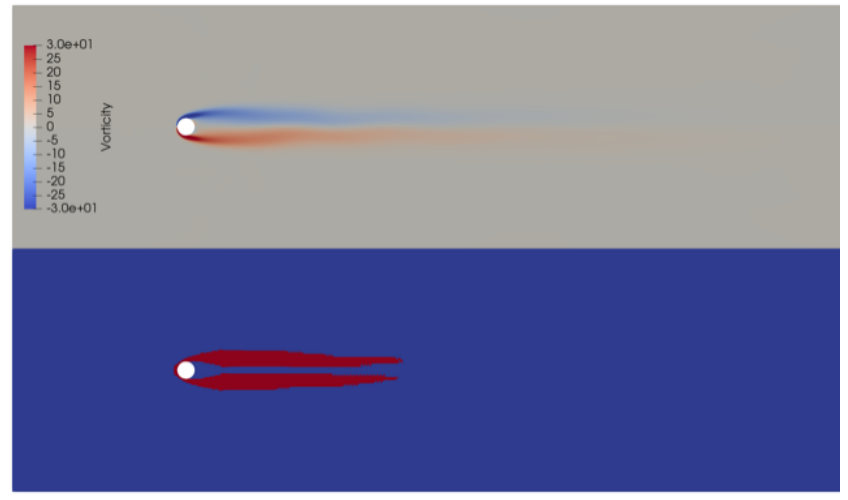

(b) $t=0.37$

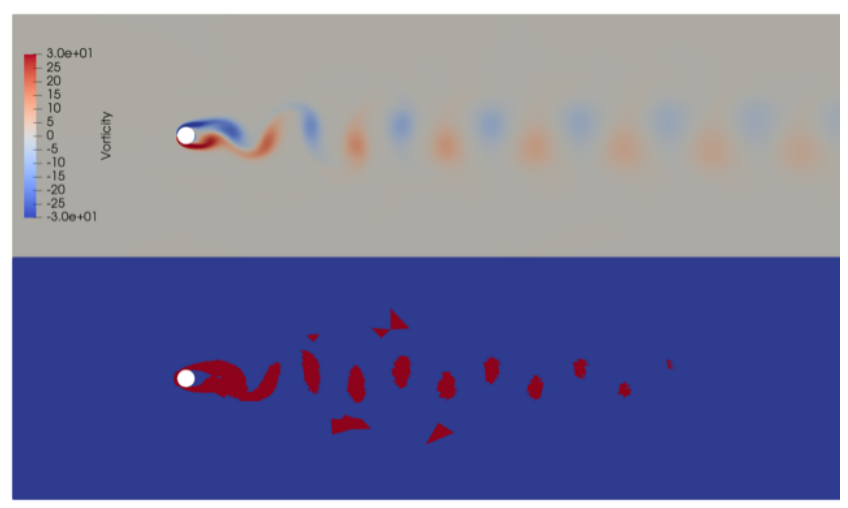

(d) $t=1.5$

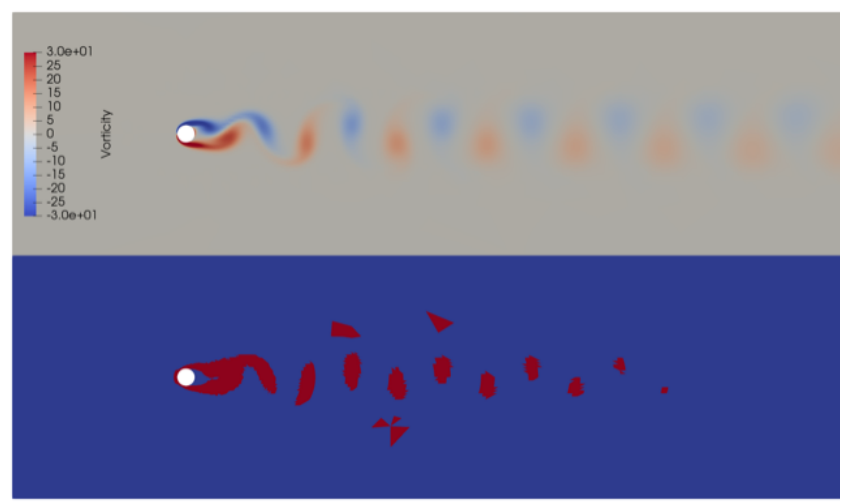

(f) $t=6.02$

6.5. Test 4 : The Navier-Stokes-Fourier system. In this test we detail the application of full spatialmodel adaptivity to the scenarios of classical forward facing step problem as well as fluid flow around an aerofoil. We simulate the Navier-Stokes-Fourier system which is given by seeking $\left(\rho_{\mu}, \rho_{\mu} \mathbf{v}_{\mu}, e_{\mu}\right)$ such that

$$
\begin{aligned}
\partial_{t} \rho_{\mu}+\operatorname{div}\left(\rho_{\mu} \mathbf{v}_{\mu}\right) & =0 \\
\partial_{t}\left(\rho_{\mu} \mathbf{v}_{\mu}\right)+\operatorname{div}\left(\rho_{\mu} \mathbf{v}_{\mu} \otimes \mathbf{v}_{\mu}\right)+\nabla p & =\operatorname{div}\left(\mu \nabla \mathbf{v}_{\mu}\right) \\
\partial_{t} e_{\mu}+\operatorname{div}\left(\left(e_{\mu}+p\right) \mathbf{v}_{\mu}\right) & =\operatorname{div}\left(\mu\left(\nabla \mathbf{v}_{\mu}\right) \cdot \mathbf{v}_{\mu}+\mu \nabla T_{\mu}\right) \\
24 &
\end{aligned}
$$


Figure 6. A numerical experiment testing model adaptivity on the Isothermal NavierStokes system. The simulation is described in $\S 6.4$. Here we display the error induced through the model adaptive strategy. We plot $\left|\boldsymbol{w}_{h}-\boldsymbol{w}_{\mu, h}\right|$, that is, the difference between the approximation of the full expensive model and that of the adaptive approximation. Note that $\left\|\boldsymbol{w}_{\mu, h}\right\|_{\mathrm{L}_{\infty}} \approx 1.7$ hence the relative error at final time is around $15 \%$.

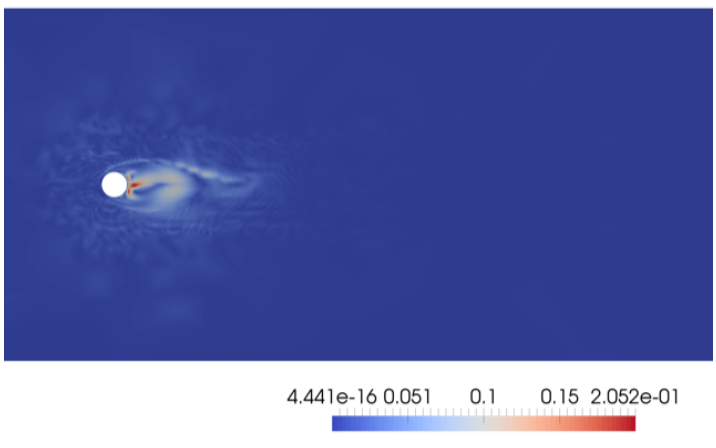

(a) $t=0.04$

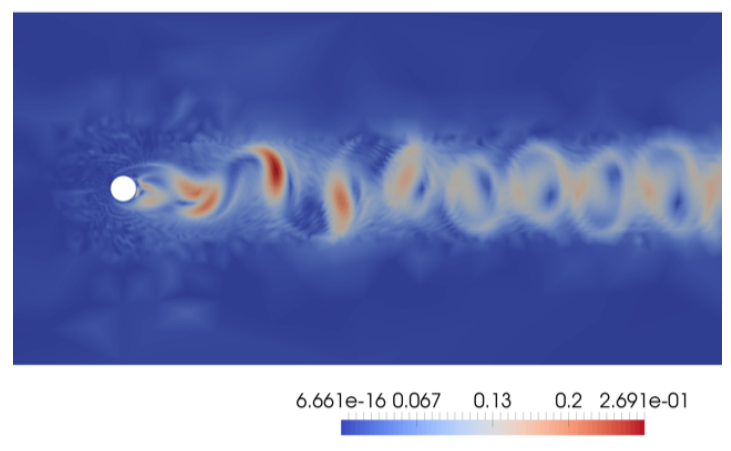

(b) $t=6.02$

in a supersonic regime. In this case our "complex" model is given by (6.20) and the approximation is given by

$$
\begin{aligned}
\partial_{t} \rho+\operatorname{div}(\rho \mathbf{v}) & =0 \\
\partial_{t}(\rho \mathbf{v})+\operatorname{div}(\rho \mathbf{v} \otimes \mathbf{v})+\nabla p & =0 \\
\partial_{t} e+\operatorname{div}((e+p) \mathbf{v}) & =0 .
\end{aligned}
$$

The specific parameters are detailed in (3.3). As in Test 3 we can rewrite this as a system with $\boldsymbol{w}=$ $\left(\rho_{\mu}, \rho_{\mu} \mathbf{v}_{\mu}, e_{\mu}\right)$ for the unknowns and (6.11) becomes

$$
\partial_{t} \boldsymbol{w}+\operatorname{div}(\boldsymbol{f}(\boldsymbol{w}))=\boldsymbol{D}(\boldsymbol{w}),
$$

with

$$
\boldsymbol{D}(\boldsymbol{w})=\left[\begin{array}{c}
0 \\
\operatorname{div}\left(\mu \nabla \mathbf{v}_{\mu}\right) \\
\operatorname{div}\left(\mu\left(\nabla \mathbf{v}_{\mu}\right) \cdot \mathbf{v}_{\mu}+\mu \nabla T_{\mu}\right) .
\end{array}\right]
$$

representing the diffusion term. We choose a piecewise linear $\mathrm{dG}$ scheme similar to that in previous tests of the form

$$
\begin{aligned}
\int_{\mathscr{T}} \partial_{t} \boldsymbol{w}_{h} \cdot \boldsymbol{\phi}-\boldsymbol{f}\left(\boldsymbol{w}_{h}\right) \cdot \nabla \boldsymbol{\phi}+\int_{\mathscr{E}} \boldsymbol{F}\left(\boldsymbol{w}_{h}^{-}, \boldsymbol{w}_{h}^{+}\right) \llbracket \boldsymbol{\phi} \rrbracket & =\mathscr{D}\left(\boldsymbol{w}_{h}, \boldsymbol{\phi} ; \widehat{\mu}\right) \quad \text { for all } \boldsymbol{\phi} \in \mathbb{V}_{q}, \\
u_{h}(0) & =\mathscr{P}_{q}\left[u_{0}\right]
\end{aligned}
$$

where, in $2 d$,

$$
\mathscr{f}(\boldsymbol{w})=\left[\begin{array}{cc}
\rho v_{1} & \rho v_{2} \\
\rho v_{1}^{2}+p & \rho v_{1} v_{2} \\
\rho v_{2} v_{1} & \rho v_{2}^{2}+p \\
(e+p) v_{1} & (e+p) v_{2}
\end{array}\right],
$$

We make use of a 3rd order strong stability preserving Runge-Kutta IMEX scheme for the temporal discretisation, a piecewise linear dG scheme with the Richtmyer fluxes (6.3) and an IP discretisation of both dissipative terms. Note that in these experiments we do not compute the difference between the adaptive approximation and the "complex model". The reason is that a fair and accurate comparison would require the simulation of the full INS system on a uniform mesh of mesh width matching the smallest mesh width in the adaptive approximation. This is computationally unfeasible. 
The algorithm we used to simulate this problem is given in pseudocode below. This is a maximum type strategy where elements with the largest local error in space, determined by the indicator $\mathscr{E}_{D}$ are refined. In addition we solve the full NSF system over those elements with largest modelling error, determined by the indictor $\mathscr{E}_{M}$.

\subsection{Spatial-Model Adaptation.}

Require: $\left(\tau, t_{0}, T, \boldsymbol{w}^{0}\right.$, tol, $\left.\mathrm{tol}_{c}, \mu\right)$

Ensure: $\left(\boldsymbol{w}_{h}^{n}\right)_{n=1, \ldots, N}$, spatial-model adaptive solution

$\widehat{\mu}(x, 0):=0$

$\triangleright$ Initialise parameters

$t=t_{0}+\tau, n=1$

while $t \leq T$ do

$\triangleright$ Loop in time

$\left(\boldsymbol{w}_{h}^{n}\right):=$ Solve one timestep of dg scheme $\left(\boldsymbol{w}_{h}^{n-1}, \widehat{\mu}\right)$

Compute $\mathscr{E}_{D}$ and $\mathscr{E}_{M}$

for $K \in \mathscr{T}$ do

if $\left.\mathscr{E}_{M}\right|_{K}<\left.\operatorname{tol}_{C} \max _{J \in \mathscr{T}} \mathscr{E}_{M}\right|_{J}$ then $\left.\widehat{\mu}(x, t)\right|_{K}=0$

end if

if $\left.\mathscr{E}_{D}\right|_{K}<\left.\operatorname{tol}_{C} \max _{J \in \mathscr{T}} \mathscr{E}_{D}\right|_{J}$ then

Mark $K$ for coarsening

end if

end for

Coarsen marked elements

Recompute $\mathscr{E}_{D}$ and $\mathscr{E}_{M}$

for $K \in \mathscr{T}$ do

if $\left.\mathscr{E}_{M}\right|_{K} \geq$ tol $\left.\max _{J \in \mathscr{T}} \mathscr{E}_{M}\right|_{J}$ then $\left.\widehat{\mu}(x, t)\right|_{K}=\mu$

end if

if $\left.\mathscr{E}_{D}\right|_{K} \geq$ tol $\left.\max _{J \in \mathscr{T}} \mathscr{E}_{D}\right|_{J}$ then

Mark $K$ for refinement

end if

end for

Refine marked elements

$t:=t+\tau, n:=n+1$

end while

return $\left(\boldsymbol{w}_{h}^{n}\right)_{n=1, \ldots, N}$,

Remark 6.2 (Comparing the two model adaptive strategies). The strategy proposed in Algorithm 6.6 has a distinct disadvantage over Algorithm 6.1 since the "complex" model will be solved on a portion of the domain from the very beginning of the simulation. Consider, for example, Tests 1 and 2 where the solution remains smooth for some time. Applying Algorithm 6.6 to these problems results in the model adaptivity being switched on before it is really needed. For these supersonic NSF simulations the solution is nonsmooth from the very beginning of the simulation and linking model adaptivity with mesh refinement is done quite simply with the implementation of Algorithm 6.6 with successful results as can be seen from Figures 7 and 8.

Note also that a feature of both strategies, due to the form of the estimator $\mathscr{E}_{M}$, is that in the scalar case the "model coarsening" step results in all cells being coarsened and then new cells selected for "model refinement" immediately afterwards. This ensures that the complex model is only used on cells where it is truly needed.

Forward step parameters. The numerical domain we use for the forward step problem is the $[0,0] \times$ $[3 / 2,3 / 4] /[1 / 2,0] \times[3 / 2,1 / 4]$. We select $\mu=10^{-6}$. We produce a uniform initial mesh with $h=0.01$ and allow a maximum refinement level of 2 . This means the minimum meshsize through the whole simulation is $h=0.0025$. We take a uniform timestep of $\tau \approx 0.00062$. Adaptation is described with Algorithm 6.6. We take initial conditions

$$
\rho(\boldsymbol{x}, 0)=1.4 \quad \mathbf{v}(\boldsymbol{x}, 0)=(3,0)^{\boldsymbol{\top}} \quad e(\boldsymbol{x}, 0)=8
$$

and enforce boundary conditions that match the initial conditions on the left boundary. We enforce slip boundary conditions on the top and bottom boundaries and an outflow boundary condition on the right 
boundary. With the speed of sound and Mach number defined as

$$
a=\sqrt{\left|\frac{\gamma p}{\rho}\right|} \quad M=\frac{|\mathbf{v}|}{a},
$$

respectively, we compute that for this experiment $a \approx 0.82$ and hence $M \approx 3.66$.

We use Algorithm 6.6 with tol $=0.5$ and tol $_{c}=0.1$. Figure 7 gives some snapshots of the solution, the model adaptivity parameter and the underlying mesh at various times.

Aerofoil parameters. We used data from the Langely Research Center website to construct a NACA 0012 aerofoil in a box $[-1 / 2,-2] \times[3,2]$. The aerofoil leading edge is situated at the origin and tail is at $[1,0]$. We select $\mu=10^{-6}$. We produce a quasiuniform initial mesh with $h \approx 0.1$ and allow a maximum refinement level of 4 . This means the minimum meshsize throughout the whole simulation is $h \approx 0.00625$. Note that due to the shape of the aerofoil the mesh is not Cartesian. This means the reconstruction proposed in $\S 5.3$ is not valid. To compute an approximation to this reconstruction we overlay a Cartesian mesh and take the nodal values as an average of the element (or elements) containing this node. We take a uniform timestep of $\tau \approx 0.0018$. Adaptation is described with Algorithm 6.1. We take initial conditions

$$
\rho(\boldsymbol{x}, 0)=1 \quad \mathbf{v}(\boldsymbol{x}, 0)=(3 / 2,0)^{\boldsymbol{\top}} \quad e(\boldsymbol{x}, 0)=5
$$

and enforce boundary conditions that match the initial conditions on the left boundary. We enforce slip boundary conditions on the top and bottom boundaries as well as on the aerofoil and an outflow boundary condition on the right boundary. Using (6.28) we compute $a \approx 1.47$ and $M \approx 1.02$.

We use Algorithm 6.6 with tol $=0.5$ and tol $_{c}=0.1$. Figure 8 gives some snapshots of the solution, the model adaptivity parameter and the underlying mesh at various times.

Remark 6.3 (Coupling of viscosity and heat conduction). In our experiment we choose $\mu$ as both the coefficient of viscosity and the heat conduction. In practical situations these parameters may scale differently and by splitting the adaptation estimator the model adaptivity can be conducted independently for both the viscous and heat conduction term. We will not persue this here.

\section{REFERENCES}

[AGQ06] Valery Agoshkov, Paola Gervasio, and Alfio Quarteroni. Optimal control in heterogeneous domain decomposition methods for advection-diffusion equations. Mediterranean Journal of Mathematics, 3(2):147-176, 2006.

[AP93] Yves Achdou and Olivier Pironneau. The $\chi$-method for the Navier-Stokes equations. IMA J. Numer. Anal., 13(4):537-558, 1993.

[ASS99] Ricardo L. Actis, Barna A. Szabo, and Christoph Schwab. Hierarchic models for laminated plates and shells. Comput. Methods Appl. Mech. Engrg., 172(1-4):79-107, 1999.

[BBRR07] R. Becker, M. Braack, R. Rannacher, and T. Richter. Mesh and model adaptivity for flow problems. In Reactive flows, diffusion and transport, pages 47-75. Springer, Berlin, 2007.

[BCR89] Franco Brezzi, Claudio Canuto, and Alessandro Russo. A self-adaptive formulation for the Euler/Navier-Stokes coupling. Comput. Methods Appl. Mech. Engrg., 73(3):317-330, 1989.

[BE03] Malte Braack and Alexandre Ern. A posteriori control of modeling errors and discretization errors. Multiscale Model. Simul., 1(2):221-238 (electronic), 2003.

[BE04] Malte Braack and Alexandre Ern. Coupling multimodelling with local mesh refinement for the numerical computation of laminar flames. Combust. Theory Model., 8(4):771-788, 2004.

[BHR11] Michel Borrel, Laurence Halpern, and Juliette Ryan. Euler - Navier-Stokes coupling for aeroacoustics problems. In Alexander Kuzmin, editor, Computational Fluid Dynamics 2010: Proceedings of the Sixth International Conference on Computational Fluid Dynamics, ICCFD6, St Petersburg, Russia, on July 12-16, 2010, pages 427-433. Springer Berlin Heidelberg, 2011.

[CBvB05] J. M. Cnossen, H. Bijl, and E. H. van Brummelen. Model-error estimation for goal-oriented model adaptation in flow-simulations. In Finite volumes for complex applications IV, pages 173-183. ISTE, London, 2005.

$\left[\mathrm{CCG}^{+} 16\right]$ Clément Cancès, Frédéric Coquel, Edwige Godlewski, Hélène Mathis, and Nicolas Seguin. Error analysis of a dynamic model adaptation procedure for nonlinear hyperbolic equations. Commun. Math. Sci., 14(1):1-30, 2016.

[CW01] Cristian A. Coclici and Wolfgang L. Wendland. Analysis of a heterogeneous domain decomposition for compressible viscous flow. Math. Models Methods Appl. Sci., 11(4):565-599, 2001.

[DG15] Andreas Dedner and Jan Giesselmann. A posteriori analysis of fully discrete method of lines dg schemes for systems of conservation laws. Technical report, 2015.

[DGQ12] Marco Discacciati, Paola Gervasio, and Alfio Quarteroni. Heterogeneous mathematical models in fluid dynamics and associated solution algorithms. In Multiscale and adaptivity: modeling, numerics and applications, volume 2040 of Lecture Notes in Math., pages 57-123. Springer, Heidelberg, 2012.

[DPE12] Daniele Antonio Di Pietro and Alexandre Ern. Mathematical aspects of discontinuous Galerkin methods, volume 69 of Mathématiques 85 Applications (Berlin) [Mathematics 85 Applications]. Springer, Heidelberg, 2012.

[Fis15] Julian Fischer. A posteriori modeling error estimates for the assumption of perfect incompressibility in the NavierStokes equation. SIAM J. Numer. Anal., 53(5):2178-2205, 2015. 
[FJN12] Eduard Feireisl, Bum Ja Jin, and Antonín Novotný. Relative entropies, suitable weak solutions, and weak-strong uniqueness for the compressible Navier-Stokes system. J. Math. Fluid Mech., 14(4):717-730, 2012.

[FNS11] Eduard Feireisl, Antonín Novotný, and Yongzhong Sun. Suitable weak solutions to the Navier-Stokes equations of compressible viscous fluids. Indiana Univ. Math. J., 60(2):611-631, 2011.

[GHM14] Emmanuil H. Georgoulis, Edward Hall, and Charalambos Makridakis. A posteriori error control for discontinuous galerkin methods for first order hyperbolic problems. Recent Developments in Discontinuous Galerkin Finite Element Methods for Partial Differential Equations: 2012 John H Barrett Memorial Lectures, 2014.

[GHM16] Martin J. Gander, Laurence Halpern, and Véronique Martin. A new algorithm based on factorization for heterogeneous domain decomposition. Numerical Algorithms, pages 1-29, 2016.

[GMP15] Jan Giesselmann, Charalambos Makridakis, and Tristan Pryer. A posteriori analysis of discontinuous galerkin schemes for systems of hyperbolic conservation laws. accepted for publication in SIAM J. Numer. Anal., 2015.

$\left[\mathrm{HGA}^{+}{ }^{12}\right]$ Florian Hindenlang, Gregor J. Gassner, Christoph Altmann, Andrea Beck, Marc Staudenmaier, and Claus-Dieter Munz. Explicit discontinuous Galerkin methods for unsteady problems. Comput. ES Fluids, 61:86-93, 2012.

[HH02] Ralf Hartmann and Paul Houston. Adaptive discontinuous Galerkin finite element methods for nonlinear hyperbolic conservation laws. SIAM J. Sci. Comput., 24(3):979-1004 (electronic), 2002.

[HW08] Jan S. Hesthaven and Tim Warburton. Nodal discontinuous Galerkin methods, volume 54 of Texts in Applied Mathematics. Springer, New York, 2008. Algorithms, analysis, and applications.

[Kru70] S. N. Kružkov. First order quasilinear equations with several independent variables. Mat. Sb. (N.S.), 81 (123):228255, 1970.

[LT13] Corrado Lattanzio and Athanasios E. Tzavaras. Relative entropy in diffusive relaxation. SIAM J. Math. Anal., 45(3):1563-1584, 2013.

[MCGS15] Hélène Mathis, Clément Cancès, Edwige Godlewski, and Nicolas Seguin. Dynamic model adaptation for multiscale simulation of hyperbolic systems with relaxation. J. Sci. Comput., 63(3):820-861, 2015.

[MN03] Charalambos Makridakis and Ricardo H. Nochetto. Elliptic reconstruction and a posteriori error estimates for parabolic problems. SIAM J. Numer. Anal., 41(4):1585-1594, 2003.

[Nic73] K Nickel. Prandtl's boundary-layer theory from the viewpoint of a mathematician. Annual Review of Fluid Mechanics, 5(1):405-428, 1973.

[OV00] J. Tinsley Oden and Kumar S. Vemaganti. Estimation of local modeling error and goal-oriented adaptive modeling of heterogeneous materials. I. Error estimates and adaptive algorithms. J. Comput. Phys., 164(1):22-47, 2000.

[PV14] Simona Perotto and Alessandro Veneziani. Coupled model and grid adaptivity in hierarchical reduction of elliptic problems. J. Sci. Comput., 60(3):505-536, 2014.

[SO99] E. Stein and S. Ohnimus. Anisotropic discretization- and model-error estimation in solid mechanics by local Neumann problems. Comput. Methods Appl. Mech. Engrg., 176(1-4):363-385, 1999. New advances in computational methods (Cachan, 1997).

[SRO11] Erwin Stein, Marcus Rüter, and Stephan Ohnimus. Implicit upper bound error estimates for combined expansive model and discretization adaptivity. Comput. Methods Appl. Mech. Engrg., 200(37-40):2626-2638, 2011.

[USDM06] Jens Utzmann, Thomas Schwartzkopff, Michael Dumbser, and Claus-Dieter Munz. Heterogeneous domain decomposition for numerical aeroacoustics. In Multifield problems in solid and fluid mechanics, volume 28 of Lect. Notes Appl. Comput. Mech., pages 429-459. Springer, Berlin, 2006.

[VO01] Kumar S. Vemaganti and J. Tinsley Oden. Estimation of local modeling error and goal-oriented adaptive modeling of heterogeneous materials. II. A computational environment for adaptive modeling of heterogeneous elastic solids. Comput. Methods Appl. Mech. Engrg., 190(46-47):6089-6124, 2001.

[vOBPvB15] T. M. van Opstal, P. T. Bauman, S. Prudhomme, and E. H. van Brummelen. Goal-oriented model adaptivity for viscous incompressible flows. Comput. Mech., 55(6):1181-1190, 2015.

[Xu00] Chuanju Xu. An efficient method for the Navier-Stokes/Euler coupled equations via a collocation approximation. SIAM J. Numer. Anal., 38(4):1217-1242, 2000.

Jan Giesselmann

Institute of Applied Analysis and Numerical Simulation

UNIVERSity OF STUtTGART

PFAFFENWALDRING 57

D-70563 StutTGarT

GERMANY

E-mail address: jan.giesselmann@mathematik.uni-stuttgart.de

TRISTAN PRYER,

Department of Mathematics and Statistics,

UNIVERSITY OF READING,

WhiteKNIGHTS,

PO Box 220,

READING RG6 6AX,

$\mathrm{UK}$

E-mail address: T.Pryer@reading.ac.uk 
FiguRE 7. An experiment using spatial-model adaptivity on the forward facing step problem. We plot the density and the model adaptivity parameter together with the underlying mesh. Notice that the mesh correctly adapts as the shocks form and propogate and that model adaptivity is on when the solution is close to shocking and near the step on the boundary, where the solution is most singular.

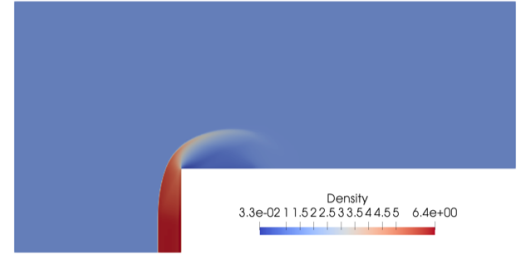

(a) Density at $t=0.0774$.

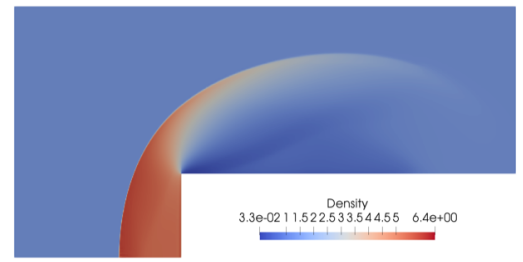

(d) Density at $t=0.2477$.

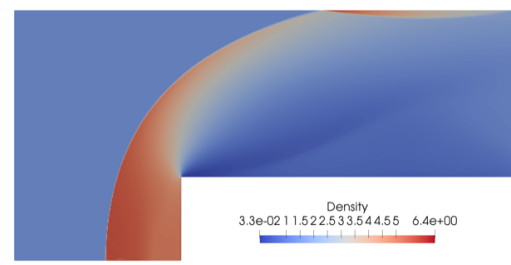

(g) Density at $t=0.3715$.

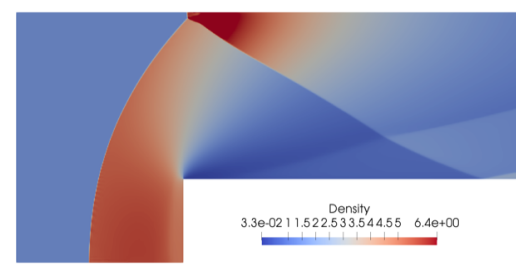

(j) Density at $t=0.743$.

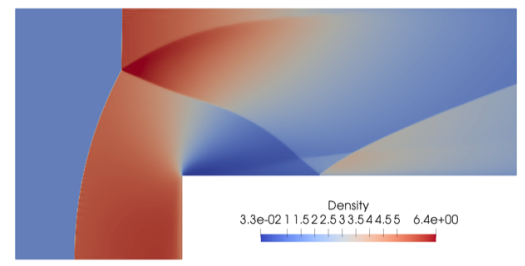

(m) Density at $t=1.2384$.

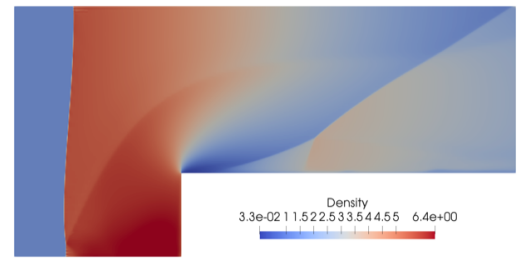

(p) Density at $t=1.8576$.

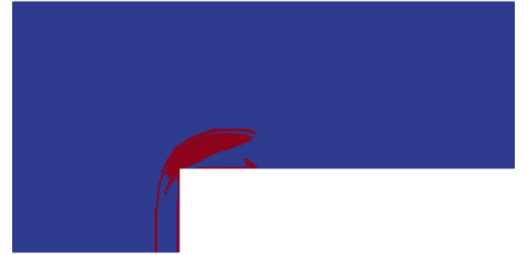

(b) $\varepsilon$ at $t=0.0774$

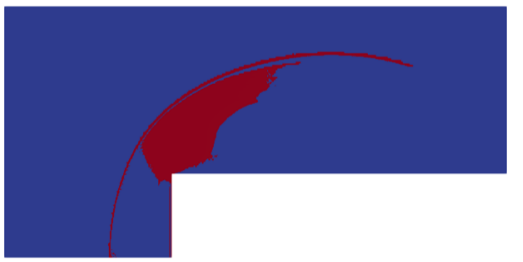

(e) $\varepsilon$ at $t=0.2477$.

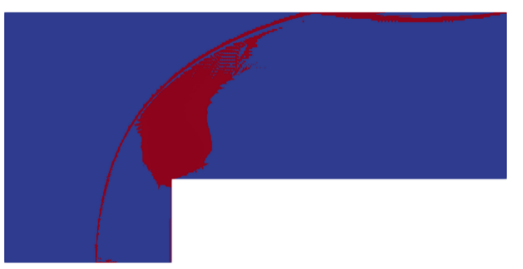

(h) $\varepsilon$ at $t=0.3715$.

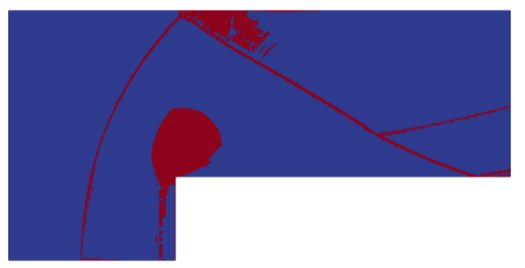

(k) $\varepsilon$ at $t=0.743$.

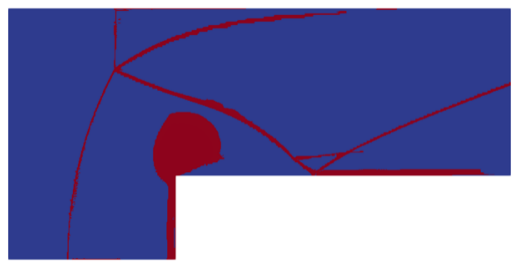

(n) $\varepsilon$ at $t=1.2384$.

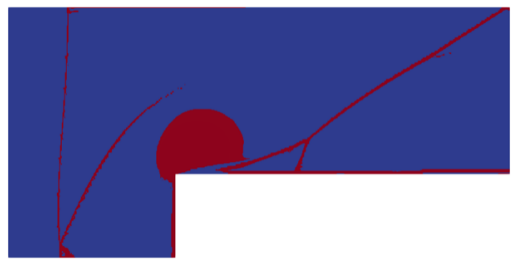

29

(q) $\varepsilon$ at $t=1.8576$.

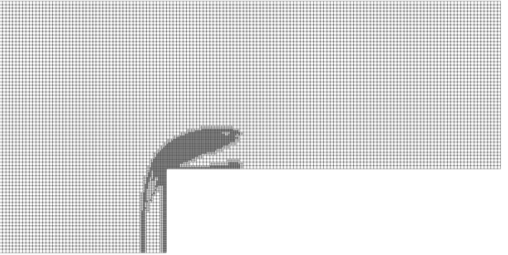

(c) Mesh at $t=0.0774$.

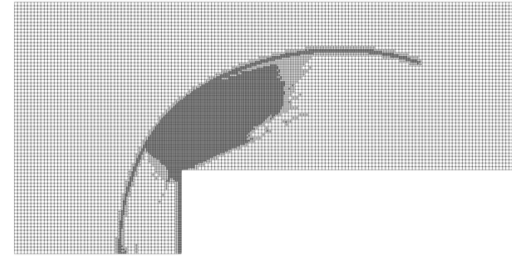

(f) Mesh at $t=0.2477$.

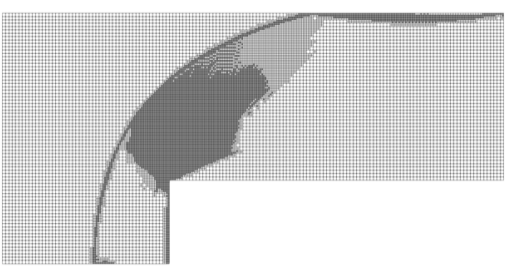

(i) Mesh at $t=0.3715$.

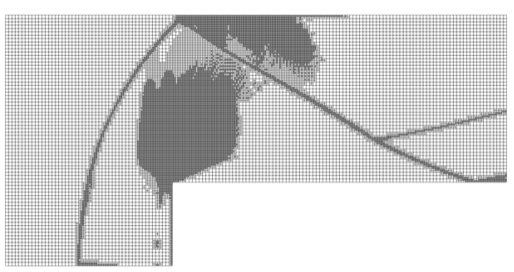

(1) Mesh at $t=0.743$.

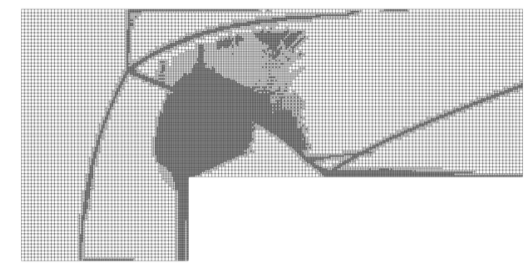

(o) Mesh at $t=1.2384$.

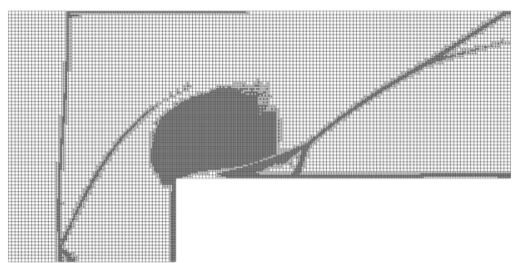

(r) Mesh at $t=1.8576$. 
FIGURE 8. An experiment using spatial-model adaptivity for flow around an aerofoil. We plot the density and the model adaptivity parameter together with the underlying mesh. Notice that the mesh correctly adapts as the shocks form and propogate and that model adaptivity is on when the solution is close to shocking.

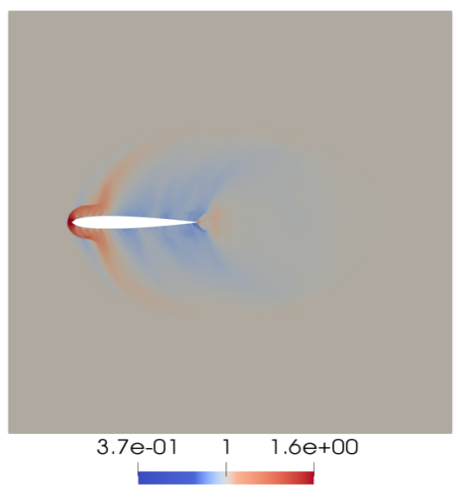

(a) Density at $t=0.0363$.

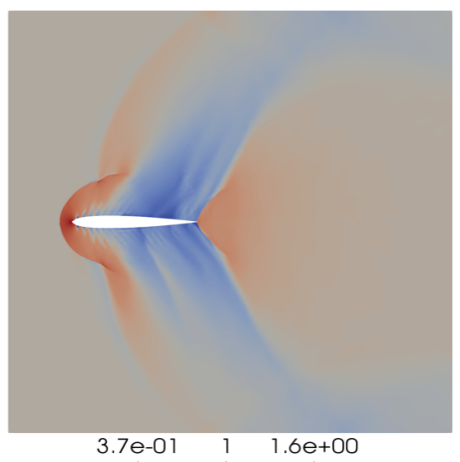

(d) Density at $t=0.127$.

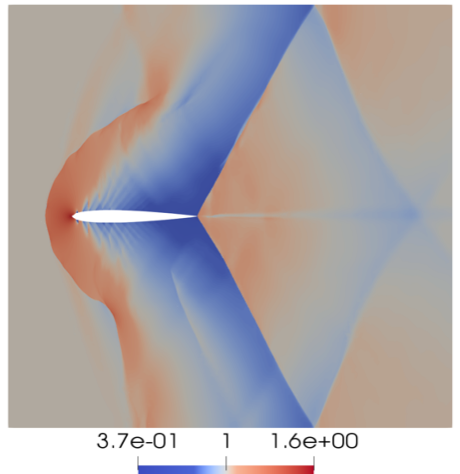

(g) Density at $t=0.363$.

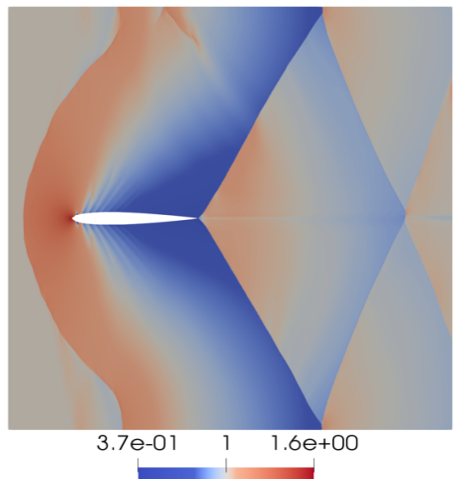

(j) Density at $t=0.9074$.

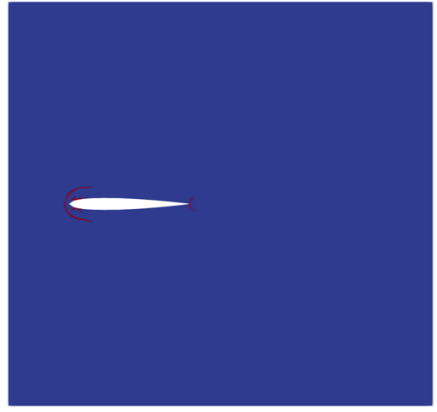

(b) $\varepsilon$ at $t=0.0363$.

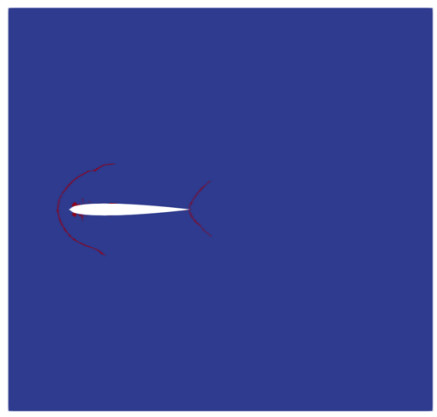

(e) $\varepsilon$ at $t=0.127$.

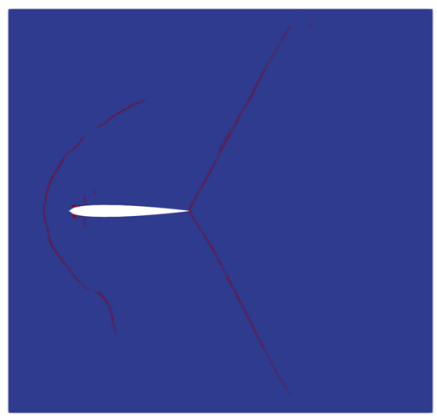

(h) $\varepsilon$ at $t=0.363$.

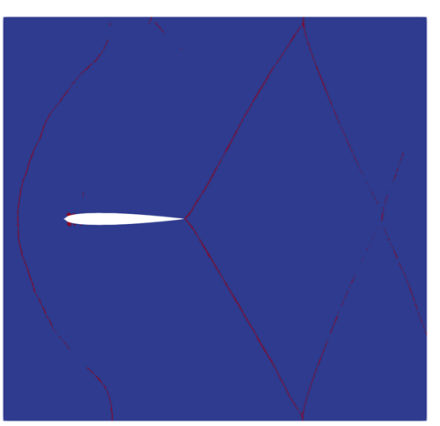

30

(k) $\varepsilon$ at $t=0.9074$.

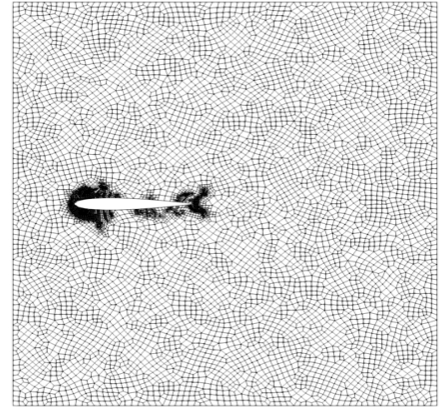

(c) Mesh at $t=0.0363$.

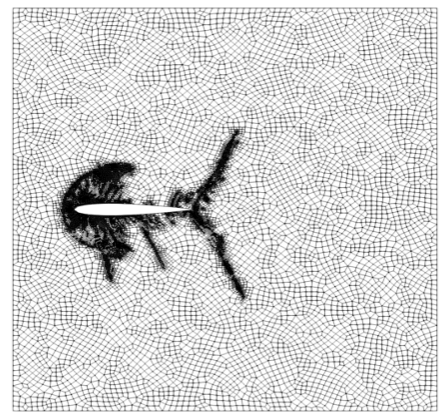

(f) Mesh at $t=0.127$.

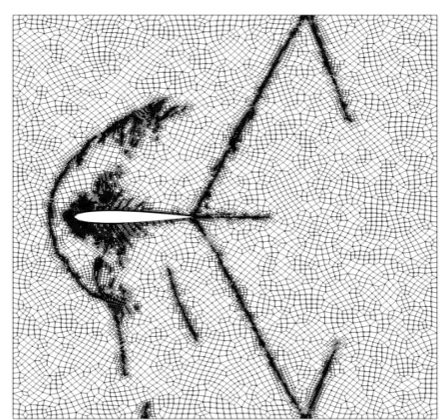

(i) Mesh at $t=0.363$.

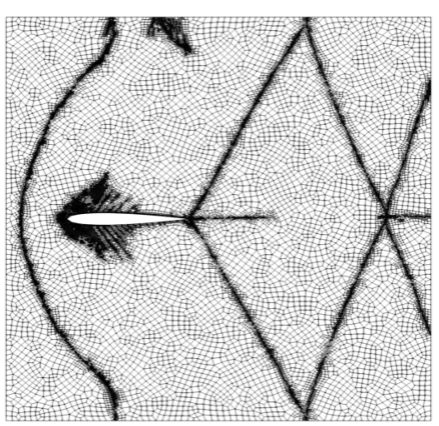

(1) Mesh at $t=0.9074$. 